\title{
ANÁLISIS FLORÍSTICO Y ESTRUCTURAL DE LOS BOSQUES PREMONTANOS EN EL MUNICIPIO DE AMALFI (ANTIOQUIA, COLOMBIA)
}

\author{
Floristic and structural analysis of premontane humid forests \\ in Amalfi (Antioquia, Colombia)
}

Palabras clave: bosques premontanos, Andes, diversidad florística, estructura de la vegetación, Amalfi, Antioquia.

Keywords: premontane forests, Andes, floristic diversity, vegetation structure, Amalfi, Antioquia.

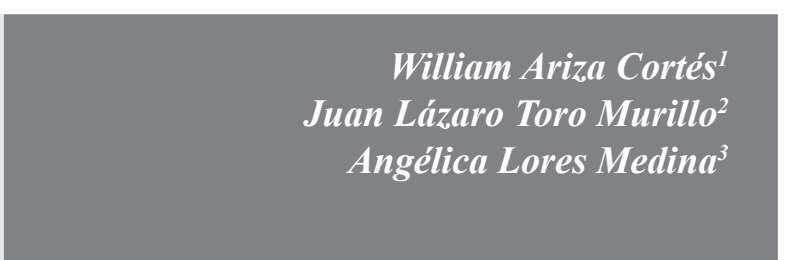

yor peso ecológico (IVI) del bosque. A pesar de la enorme diversidad documentada, en la actualidad estos bosques están sujetos a un intenso proceso de fragmentación y pérdida de cobertura.

\begin{abstract}
The floristic composition and the structure of a humid hill forest were determined. The forest is located in the northernmost area of the Colombian Central Mountain Range. The methodology proposed by ISA-JAUM was employed in this study, from a 0.1 ha sample. Moreover, plant material was collected from clear spots, stubbles and grasslands. A total of 421 vascular plants species were found. Two hundred and thirty eight of these come from the 0.1 ha sample and only 150 had DBH $>2.5 \mathrm{~cm}$, which highlights the benefits of the sample method employed, since it was possible to record a large number of epiphytic and herbaceous elements in the forest. In general, the composition matches what was previously reported in similar areas. The families Melastomataceae (32), Lauraceae (31), Rubiaceae (29) and Araceae (15) were the ones holding the largest number of species. New chorological records for the state of Antioquia were reported in this study; for instance, Colombobalanus
\end{abstract}

1 Proyecto Curricular de Ingeniería Forestal, Universidad Distrital Francisco José de Caldas. Correspondencia: arizacortes@, yahoo.com

2 Corporación Autónoma Regional del Centro de Antioquia. jltoro@corantioquia.gov.co

3 Proyecto Forestal, Instituto Colombiano Agropecuario. gelastocoridae@gmail.com 
excelsa (Fagaceae). High density of individuals (388) was found at structural level. The patterns of height and diametrical classes followed the typical distribution of disetaneous tropical forests, in which the largest amount of individuals occur in the lowest classes and few species with individuals having the greatest diameters and heights account for the major ecological weight in the forest. Despite the great diversity documented, these forests are currently subject to an intensive process of fragmentation and loss of coverage.

\section{INTRODUCCIÓN}

La región septentrional de la cordillera de los Andes es considerada uno de los lugares más diversos del planeta (Hernández \& Sánchez 1992, Gentry 1995, Cuervo 2002). Su posición estratégica y la enorme diversidad de hábitats hacen posible el establecimiento de una gran cantidad de organismos (Cavelier et al. 2001); otro factor importante en la concentración de especies en el área fueron las numerosas migraciones de taxones debido a la reconexión entre América del Sur y América del Norte durante el Plio-Pleistoceno (Webster 1995).

Dentro de los ecosistemas andinos, la franja altitudinal correspondiente a los llamados bosques subandinos o premontanos presenta algunos de los niveles más altos de concentración de especies por unidad de área, debido a la confluencia de elementos tropicales y montanos (Gentry 1982, 1995, 2001; Rangel \& Velásquez 1997). La interacción de factores como precipitación, latitud y altitud privilegiados hacen que estos bosques posean una inmensa riqueza natural. La Reserva Forestal del Bajo Cauca - Nechí, ubicada en el extremo norte de la Cordillera Central, ha sido considerada una de las zonas más diversas y con mayor cantidad de endemismos en nuestro país (Hernández \& Sánchez 1992, Cogollo \& Ramírez 1997). Una de las áreas con mayores remanentes de bosque premontano dentro de la reserva es el municipio de Amalfi, ubicado al noroeste del departamento de Antioquia.

Sin embargo, el conocimiento acerca de estos ecosistemas es incipiente: Tuberquia et al., en el año 2000, realizaron algunos muestreos en el área, bajo el marco de la educación ambiental; adicionalmente se han realizado recolecciones esporádicas de material vegetal, en prácticas académicas de biología de la Universidad de Antioquia, y Gómez (2005) realizó una caracterización florística y estructural en bosques premontanos del municipio de Anorí, donde se corrobora la alta diversidad florística de estos ecosistemas.

El presente trabajo pretende aumentar el conocimiento sobre la composición, la diversidad y la estructura de los bosques premontanos del municipio de Amalfi, constituyendo la base para formular las estrategias de manejo y conservación necesarias en estas áreas.

\section{METODOLOGÍA}

\section{ÁREA DE ESTUDIO}

El estudio se realizó en el sector norte del municipio de Amalfi (noroeste del departamento de Antioquia), en la vereda Guayabito, microcuenca de la quebrada Guayabito, localizada a $06^{\circ} 53^{\prime}$ '58' $\mathrm{N}$ y $75^{\circ} 04^{\prime} 58^{\prime \prime} \mathrm{O}$, en alturas comprendidas entre 1500 y 1800 msnm (Figura 1), sobre pendientes que oscilan entre el 60 y el 120\%. La precipitación promedio es de $3091 \mathrm{~mm}$ al año y la temperatura promedio anual es de $22^{\circ} \mathrm{C}$; de acuerdo con la clasificación de Holdridge (IGAC 1976), corresponde a la zona de vida del bosque muy húmedo premontano (bmh-PM) en transición al bosque pluvial premontano (bp-PM). Actualmente estos bosques se encuentran sometidos a una alta tasa de fragmentación y extracción maderera. 


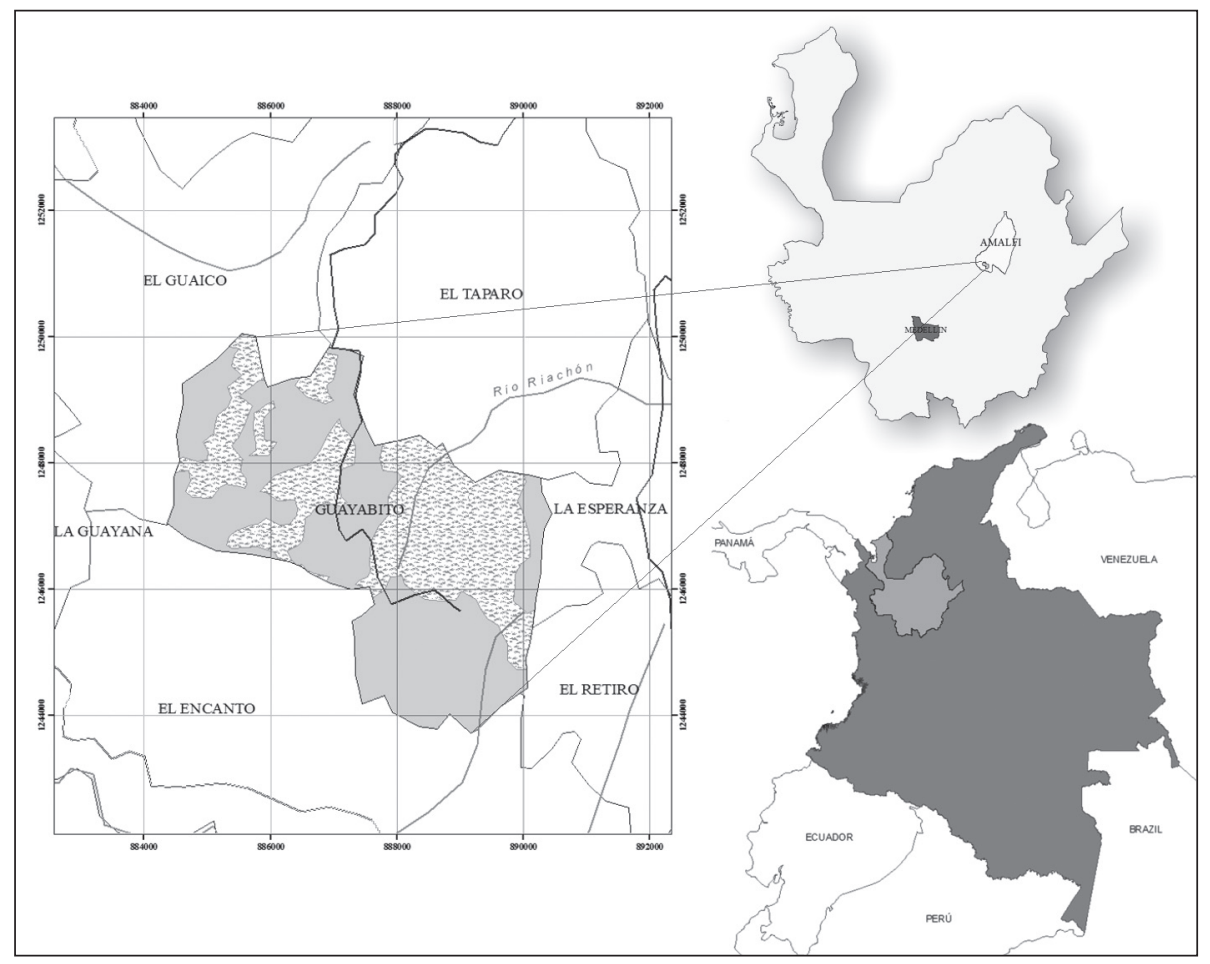

Figura 1. Área de estudio.

\section{MUESTREO}

Se establecieron cinco transectos rectangulares de $50 \times 4 \mathrm{~m}$, siguiendo la metodología propuesta por ISA-JAUM (2004), en donde se censaron y midieron todos los individuos con un diámetro a la altura del pecho (DAP) $\geq 2.5 \mathrm{~cm}$, y adicionalmente en el lado derecho del transecto se recolectaron o registraron todas las especies, como helechos, hierbas y epífitas, que por su porte o hábito de crecimiento no alcanzaban los $2.5 \mathrm{~cm}$ de DAP (Figura 2).

Las variables consideradas fueron: altura total, altura comercial, diámetro a la altura del pecho (DAP) y cobertura (proyección de las copas en los ejes $x$ y y). La clasificación de los hábitos de crecimiento se realizó de acuerdo con los lineamientos propuestos por la metodología ISA-JAUM (2004).

Se recolectaron muestras botánicas del primer individuo de cada especie, tomando como mínimo tres duplicados. Cada ejemplar botánico fue debidamente marcado con el número del levantamiento, las iniciales y el número del colector; posteriormente los ejemplares fueron procesados de acuerdo con los lineamientos propuestos por Ariza \& Medina (2006). En lo posible, cada individuo recolectado fue fotografiado con el fin de facilitar el proceso de identificación.

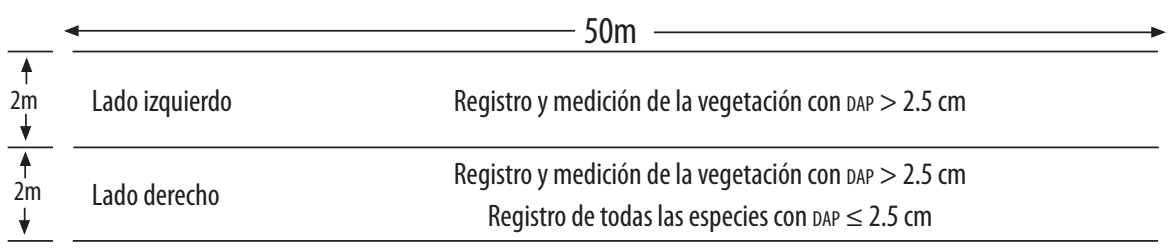

Figura 2. Esquema del censado y medición del RAP modificado (ISA-JAUM 2004). 
Adicionalmente se realizaron tres salidas de campo, durante las cuales se hicieron colecciones generales en zonas aledañas a los transectos (bordes y claros de bosque, potreros, etc.), con el fin de contar con un mayor número de muestras en estado reproductivo y facilitar la determinación del material procedente de los transectos. Los ejemplares botánicos obtenidos en el proyecto se depositaron en la colección del Herbario Nacional Colombiano (COL), Herbario de Referencia de Corantioquia (HRC) y Herbario Forestal Gilberto Emilio Mahecha Vega (UDBC), principalmente bajo la numeración del primer autor (Anexo 1).

\section{ANÁLISIS Y PROCESAMIENTO DE LA INFORMACIÓN}

El material vegetal fue procesado en el Herbario del Jardín Botánico de Medellín (JAUM); la curaduría del material se realizó empleando la información taxonómica existente para cada uno de los grupos, con la colaboración de especialistas y confrontando con las colecciones del Herbario del Jardín Botánico de Medellín y del Herbario Nacional Colombiano (COL).

Para el análisis florístico se empleó la clasificación de las familias de pteridofitos propuesta por Smith et al. (2006); las familias de angiospermas y gimnospermas fueron agrupadas de acuerdo con el sistema de clasificación de APG (2003). En el análisis fisionómico-estructural de la vegetación se calculó el Índice de Valor de Importancia (IVI) para los individuos con DAP $>2.5 \mathrm{~cm}$, y se establecieron intervalos de clases de altura y DAP con base en los valores máximos y mínimos de cada parámetro y el número de individuos (Lema 1995). En la estimación de la diversidad se calcularon los índices Margalef (Dmg), Menhinick (Dmn) Berger-Parker y Simpson, teniendo en cuenta que son los más empleados para la comparación de la diversidad alfa (Magurran 1989).

\section{RESULTADOS}

\section{COMPOSICIÓN Y RIQUEZA FLORÍSTICA}

En el muestreo de 0.1 ha se encontraron 72 familias de angiospermas, una de gimnospermas y cin- co de pteridofitos agrupadas en 163 géneros, y 238 especies en total. Las familias con mayor número de especies fueron Lauraceae (23), Rubiaceae (21), Melastomataceae (18), Araceae (14), Clusiaceae (11), Leguminosae (10), Orchidaceae (9) y Arecaceae (7). Los géneros mejor representados fueron Miconia (9), Ocotea (7), Inga y Clusia (5), Anthurium, Philodendron y Palicourea (4).

$\mathrm{Al}$ incluir solamente los individuos con DAP $>2.5 \mathrm{~cm}$ se encontraron 57 familias, 102 géneros y 150 especies, donde Lauraceae (20) y Melastomataceae (15) fueron las familias con mayor número de especies, seguidas de Clusiaceae (10), Myrtaceae (7), Leguminosae (7) y Arecaceae (6). Dentro de los géneros mejor representados se destacan Clusia (7), Inga y Myrcia (4). Las recolecciones generales realizadas en zonas de potrero, bordes de bosque y zonas de claros, aumentaron la cantidad de especies de 238 a 421; de igual manera, el número de géneros pasó de 163 a 263 y el número de familias se elevó de 72 a 104. Entre algunos de los taxones encontrados solamente con este tipo de muestreo se destacan los miembros de la familia Asteraceae, Urticaceae, Salicaceae y Loranthaceae (anexo 1). En la Tabla 1 se muestra la riqueza de algunos bosques premontanos del país a partir de muestras de 0.1 ha.

Se reportan diversas especies por primera vez para el departamento de Antioquia. Uno de los registros más sobresalientes es la especie Colombobalanus excelsa, la cual había sido reportada en los departamentos de Valle, Huila y Santander. Adicionalmente se amplía considerablemente la distribución altitudinal de numerosas especies. En la Tabla 2 se observa la ampliación en la distribución geográfica y altitudinal de algunas de las especies encontradas.

\section{ESTRUCTURA VERTICAL}

La estructura vertical indica que la mayor parte de los individuos se encuentran en los dos primeros intervalos de clase de altura $4.2<6.9 \mathrm{~m}(20.11 \%)$ y $1.5<4.2 \mathrm{~m}(18.25 \%)$. Este primer grupo de alturas corresponde a géneros como Clusia y Miconia, así como a gran cantidad de individuos de Wettinia fascicularis. La curva continúa disminuyendo progresivamente hasta la clase de altura $15<17.7$ $\mathrm{m}$, donde se eleva debido a la gran abundancia de 
individuos de especies como Compsoneura aff. capitellata y Roucheria columbiana. A partir de allí la curva decrece progresivamente hasta los estratos superiores, donde es posible encontrar individuos con alturas hasta de $29 \mathrm{~m}$, los cuales corresponden a especies como Vochysia aff. aurantiaca, Ternstroemia macrocarpa, Chrysophyllum prieurii, Aniba coto y Quercus humboldtii (Figura 3).

\section{ESTRUCTURA HORIZONTAL}

El total de individuos con DAP $>2.5$ fue de 388; el hábito predominante es el arbóreo con un $63.4 \%$ del total de los individuos, seguido del hábito arbustivo con un 10\%. Las cinco especies más abundantes en el bosque incluyen al $22.49 \%$ del total de los individuos; se destacan los valores de abundancia y frecuencia de la especie Wettinia fascicularis. Las especies más dominantes aportaron el 3623\% del área basal del muestreo $\left(5.72 \mathrm{~m}^{2}\right)$, y tan sólo dos especies se encontraron presentes en los cinco transectos realizados (Wettinia fascicularis y Roucheria columbiana). En la Tabla 3 es posible observar cómo las dos especies más importantes ecológicamente concentran casi el 25\% del IVI en los transectos (Anexo 2).

Tabla 1. Riqueza de especies y familias en algunos bosques premontanos de Colombia, a partir de muestras de 0.1 ha.

\begin{tabular}{|c|c|c|c|c|c|}
\hline \multirow[t]{2}{*}{ Fuente } & \multirow[t]{2}{*}{ Localidad } & \multirow[t]{2}{*}{ Altura (msnm) } & \multicolumn{2}{|c|}{ No. de especies } & \multirow{2}{*}{$\begin{array}{l}\text { No. de } \\
\text { familia }\end{array}$} \\
\hline & & & DAP $>0$ & $\mathrm{DAP}>2.5 \mathrm{~cm}$ & \\
\hline Cantillo \& Fajardo, 2004 & Reserva de Yotoco (Valle) & $1200-1700$ & - & 80 & 40 \\
\hline Gentry, 1988 & Reserva la Planada (Nariño) & 1800 & - & 116 & 54 \\
\hline Navarro, 2003 & Cubarral (Meta) & 1900 & & 92 & 55 \\
\hline \multirow{3}{*}{$\begin{array}{l}\text { Gómez, } 2005 \text { Anorí } \\
\text { (Antioquia) }\end{array}$} & $\begin{array}{c}\text { Vereda El Roble, Reserva La } \\
\text { Forzosa }\end{array}$ & $1530-1560$ & 148 & 145 & 51 \\
\hline & $\begin{array}{c}\text { Vereda El Roble, Reserva La } \\
\text { Forzosa }\end{array}$ & $1620-1765$ & 201 & 133 & 60 \\
\hline & Vereda Santa Gertrudis & $1325-1460$ & 169 & 121 & 51 \\
\hline Gentry, 1995 & Antadó (Antioquia) & 1560 & - & 160 & 55 \\
\hline \multirow{2}{*}{ Varias localidades } & Napo (Ecuador) & 1150 & - & 151 & 56 \\
\hline & Río Candamo (Perú) & 800 & - & 232 & 65 \\
\hline \multicolumn{6}{|l|}{ Amalfi (Antioquia) } \\
\hline Presente estudio & Vereda Guayabito & $1500-1800$ & 238 & 150 & 72 \\
\hline
\end{tabular}

Tabla 2. Ampliación geográfica y altitudinal de la distribución de algunas especies encontradas en un bosque premontano del municipio de Amalfi.

\begin{tabular}{ccc}
\hline Especies & Distribución geográfica (Colombia) & Distribución altitudinal \\
\hline Ormosia revoluta & Valle, Antioquia* & $1600-2200$ \\
Ormosia cuatrecasasii & Chocó, Valle, Antioquia* & $50-200,1600^{*}$ \\
Marcgravia dressleri & Santander, Antioquia* & $1200-1700$ \\
Colombobalanus excelsa & Huila, Santander, Valle, Antioquia* & $1300-2300$ \\
Micropholis crotonoides & Caquetá, Chocó, Magdalena, Valle, Antioquia* & $50-1800$ \\
\hline
\end{tabular}




\begin{tabular}{ccc}
\hline Especies & Distribución geográfica (Colombia) & Distribución altitudinal \\
\hline Ecclinusa bullata & Chocó, Valle, Antioquia* & $50-1200,1800^{*}$ \\
Roucheria columbiana & Valle, Chocó, Antioquia & $50-1000,1800^{*}$ \\
Licania octandra & Amazonas, Antioquia, Caquetá, Chocó, Córdoba, Meta, & $100-800,1800^{*}$ \\
& Valle, Putumayo, Santander, Antioquia & \\
\hline
\end{tabular}

* Ampliación de la distribución a partir del presente estudio.

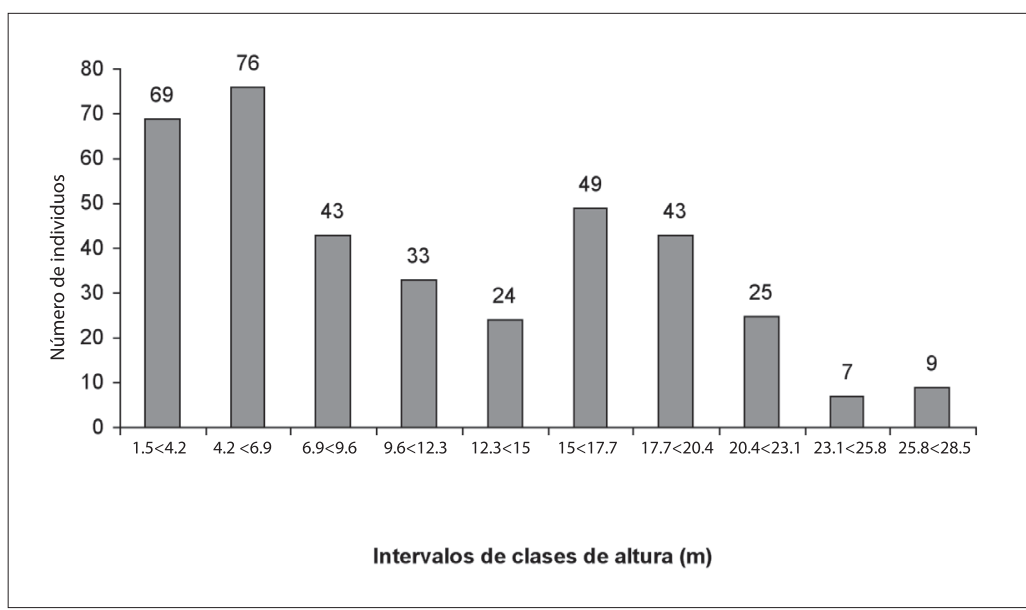

Figura 3. Estructura vertical de los individuos con DAP $>2.5 \mathrm{~cm}$ en 0.1 ha de un bosque premontano en el municipio de Amalfi, Antioquia, Colombia.

\section{ESTRUCTURA TOTAL}

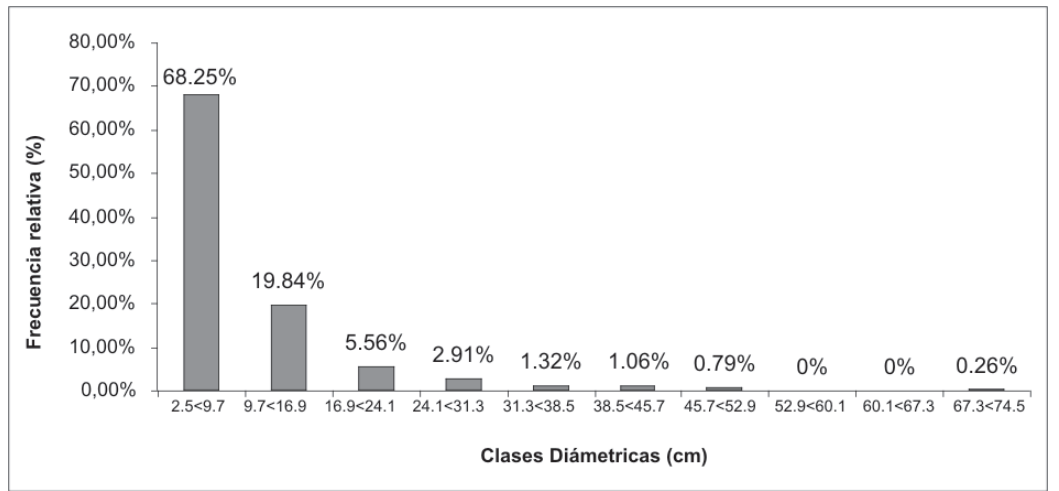

Figura 4. Distribución diamétrica de individuos con DAP $>2.5 \mathrm{~cm}$ en 0.1 ha de un bosque premontano en el municipio de Amalfi, Antioquia. 
Tabla 3. Especies con mayor valor de importancia (IVI), abundancia, frecuencia y dominancia relativas en un bosque premontano en el municipio de Amalfi (Antioquia).

\begin{tabular}{|c|c|c|}
\hline & Especie & $\begin{array}{c}\text { Valor } \\
(\%)\end{array}$ \\
\hline \multirow{6}{*}{ 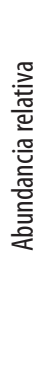 } & Wettinia fascicularis & 6.11 \\
\hline & Protium aff. tovarense & 5.38 \\
\hline & Roucheria columbiana & 4.65 \\
\hline & Miconia punctata & 3.42 \\
\hline & Compsoneura aff. capitellata & 2.93 \\
\hline & Wettinia fascicularis & 2.13 \\
\hline \multirow{7}{*}{ 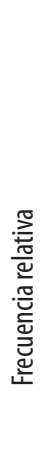 } & Roucheria columbiana & 2.13 \\
\hline & Tovomita weddelliana & 1.7 \\
\hline & Protium aff. tovarense & 1.7 \\
\hline & Compsoneura aff. capitellata & 1.7 \\
\hline & Graffenrieda latifolia & 1.7 \\
\hline & Clusia cf. magnifolia & 1.7 \\
\hline & Miconia lamprophylla & 1.7 \\
\hline \multirow{13}{*}{ 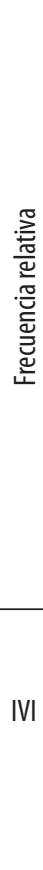 } & Miconia punctata & 1.7 \\
\hline & Virola macrocarpa & 1.7 \\
\hline & Wettinia kalbreyeri & 1.7 \\
\hline & Quercus humboldtii & 12.59 \\
\hline & Chrysophyllum prieurii & 8.06 \\
\hline & Dacryodes sp. & 6.46 \\
\hline & Ternstroemia sp. & 5.22 \\
\hline & Protium aff. tovarense & 3.89 \\
\hline & Quercus humboldtii & 13.99 \\
\hline & Protium aff. tovarense & 10.98 \\
\hline & Wettinia fascicularis & 10.86 \\
\hline & Roucheria columbiana & 10.2 \\
\hline & Chrysophyllum prieurii & 10.07 \\
\hline
\end{tabular}

Como se observa en la Figura 4 (p.86), la distribución diamétrica presentó un comportamiento en forma de "jota" invertida, encontrándose en la categoría más baja $(2.5<9.7)$ el mayor número de individuos (258), disminuyendo progresivamente en número a medida que se incrementan los valores del diámetro; no se presentaron individuos en las clases entre $52.9<67.3$ y sólo un individuo en la última clase $(67.3<74.5)$. Las especies con mayores valores de DAP fueron Quercus humboldtii $(70.66 \mathrm{~cm})$, Chrysophyllum prieurii $(49.33 \mathrm{~cm})$, Vochysia aff. aurantiaca $(47.42 \mathrm{~cm})$ y Ternstroemia macrocarpa $(46.47 \mathrm{~cm})$.

\section{DIVERSIDAD}

Los índices de diversidad de Shannon y Simpson muestran que el bosque es heterogéneo y no hay predominio de ninguna especie, como lo confirma el índice de Berger-Parker para la especie más abundante (Protium aff. tovarense) (ver Tabla 4).

Tabla 4. Índices de alfa diversidad.

\begin{tabular}{ll}
\hline \multicolumn{1}{c}{ Índice } & Valor \\
\hline Margalef & 6.735 \\
Menhinick & 3.343 \\
Berger-Parker & $* 0.06$ \\
Shannon & 5.02 \\
Uniformidad de Shannon & 0.96 \\
Simpson & 0.016 \\
\hline
\end{tabular}

*Calculado para la especie más abundante.

\section{DISCUSIÓN}

\section{RIQUEZA Y COMPOSICIÓN}

Al comparar los valores en muestreos con DAP $>2.5 \mathrm{~cm}$, el área de estudio posee una de las más altas riquezas de familias y especies documentadas en muestreos de 0.1 ha, sólo comparable con muestreos como el de Gómez (2005) en el municipio de Anorí, o los de Gentry (2001) en Antadó, el Napo en Ecuador o el río Candamo en Perú, donde la riqueza fue superior a 150 especies. La composición florística a nivel de familias es congruente con los datos obtenidos por diversos autores para la franja premontana de los Andes, con Lauraceae, Melastomoataceae y Rubiaceae como las familias más abundantes (Rangel 1995, Gentry 1995, Cavelier et al. 2001); sin embargo, en el 
presente estudio la familia Araceae aparece como una de las mejor representadas, lo que evidencia la gran abundancia y diversidad de epífitas. Existe una buena correspondencia entre los géneros y las familias más abundantes, géneros como Miconia, Anthurium e Inga ya habían sido reportados como los más abundantes en la franja premontana de los Andes, sin embargo, el número promedio de especies en estudios previos es ostensiblemente menor (Gentry 1995, Franco et al. 1997).

Al excluir del análisis los individuos con DAP $<2.5$ cm ocurre una disminución del $21 \%$ en la cantidad de familias, del $37 \%$ en la cantidad de géneros y del $36 \%$ en el número de especies, lo que evidencia la gran cantidad de elementos no leñosos dentro del bosque; la familia Araceae desaparece y familias como Myrtaceae ingresan dentro de las más abundantes, lo que muestra el efecto del diámetro mínimo sobre los resultados obtenidos en composición y riqueza. Otros autores han discutido sobre las modificaciones al muestreo tipo RAP (Rapid Assessment Plots) y la influencia del diámetro mínimo de muestreo sobre la estimación de la diversidad vegetal de los bosques tropicales (Franco et al. 1997, ISA-JAUM 2004, Dueñas et al. 2007).

Las recolecciones generales aumentan la riqueza florística en un $76 \%$, y garantizan un mayor grado de certeza en las determinaciones, por tratarse de ejemplares en estado reproductivo. Se encontró gran cantidad de elementos heliófitos, típicos de áreas abiertas y bordes de bosque, entre los que se destacan Cecropia, Pourouma, Piptocoma, Vernonathura y Munozia, entre otros. Esto evidencia el sesgo que se genera al emplear sólo este tipo de muestreo en el estudio de la diversidad florística de un ecosistema boscoso.

\section{ESTRUCTURA}

En los bosques tropicales andinos generalmente se observa una disminución progresiva en el número de individuos a medida que aumenta la altura de los árboles (Dueñas et al. 2007, Cantillo et al. 2004), sin embargo, las diferencias observadas en la distribución vertical suponen una estructura variable que puede responder a la gran dinámica de claros en ecosistemas montañosos con altas pen- dientes (Robert 2003) y a las marcadas diferencias florísticas entre los estratos.

La distribución de las clases diamétricas, que coincide con otros estudios en bosques andinos (López et al. 2006, Dueñas et al. 2007, Cantillo et al. 2004), se acerca a una distribución normal típica de bosques naturales disetáneos de poca intervención (Lamprecht 1990), debido a la alta presencia de regeneración natural, y a los pocos individuos en las clases mayores, de especies como Quercus humboldtii y Vochysia aff. aurantiaca, coincidiendo con lo reportado en el municipio de Anorí (Gómez 2005, López et al. 2006).

La importancia ecológica de las especies, representada por los valores de IVI, sugieren un comportamiento heterogéneo dentro del ecosistema. El IVI se ve fuertemente influenciado por la presencia de individuos de gran tamaño, como el caso de la especie Quercus humboldtii, la cual presentó uno de los mayores valores de IVI con tan sólo cuatro individuos, dos de los cuales poseían diámetros mayores a $45 \mathrm{~cm}$. Lo anterior coincide con datos reportados en bosques tropicales, donde unos pocos árboles con alturas y diámetros altos son los que presentan el mayor IVI dentro del bosque (Dueñas et. al 2007).

El comportamiento estructural de géneros como Wettinia, Vochysia, Protium y Compsoneura fue similar al observado por Gómez (2005) en otros bosques premontanos, donde se reportan como de alta importancia ecológica dentro del ecosistema.

\section{DIVERSIDAD}

Al comparar con otros estudios en bosques premontanos húmedos, la diversidad encontrada es notoriamente mayor (Gomez 2005, Navarro 2004)y se refleja en valores superiores de índices como los de Margalef y Menhinick, debido principalmente a la mayor cantidad de especies encontradas en el presente estudio. Al contrastar el valor obtenido en índices como los de Berger-Parker y Simpson con el listado de especies es posible observar que a pesar de la presencia de especies con alta tendencia asociativa como Quercus humboldtii la heterogeneidad del ecosistema es alta. Esto ha sido 
reportado previamente en bosques con condiciones similares de altitud y precipitación (Rangel et al. 2004, Kappelle \& Zamora 1995).

\section{CONCLUSIONES}

Los bosques premontanos presentes en la región septentrional de la Cordillera Central son ecosistemas estratégicos, con una complejidad estructural alta y una enorme diversidad florística. Así mismo, el hallazgo de numerosos registros nuevos a nivel local y regional evidencia el déficit en el muestreo de la vegetación presente en el área de estudio.

La metodología de muestreo, así como el diámetro mínimo empleado, inciden drásticamente en la estimación de la riqueza y diversidad de los bosques tropicales y especialmente en ecosistemas donde hábitos de crecimiento tales como hierbas y epífitas se encuentran bien representados. Es preciso estandarizar el diámetro mínimo de muestreo para hacer estimaciones de riqueza y diversidad representativas en cada uno de los ecosistemas estudiados.

Es necesario profundizar en el inventario de la diversidad debido a la cantidad de novedades corológicas a nivel local y regional. Adicionalmente, conviene adelantar investigaciones relacionadas con la dinámica y la funcionalidad de los bosques del área, con el fin de generar bases sólidas para el manejo y conservación de estos ecosistemas estratégicos.

\section{AGRADECIMIENTOS}

A Corantioquia por su financiación y apoyo en la elaboración del trabajo; a los funcionarios del Herbario del Jardín Botánico de Medellín (JAUM), en especial a su director Álvaro Cogollo por su apoyo constante; a los funcionarios del Herbario de la Universidad de Antioquia (HUA) y del Herbario Nacional Colombiano por su ayuda en la identificación del material botánico, y muy especialmente a Duver Botero y los propietarios de la finca Costa Rica en la vereda Guayabito por su colaboración en el trabajo de campo. A todos los que de una u otra forma colaboraron para el desarrollo de este trabajo.

\section{REFERENCIAS BIBLIOGRÁFICAS}

\section{ANGIOSPERM PHYLOGENY GROUP II.}

2003. An update of the Angiosperm Phylogeny Group classification for the orders and families of flowering plants. Bot. J. Linn. Soc. 141: 399-436.

Ariza, W. \& R. Medina. 2006. Guía para la identificación de las variedades de coca cultivadas en Colombia. Presidencia de la República, Universidad Distrital Francisco José de Caldas. Bogotá. Pg. 40.

Cantillo, E. \& A. Fajardo. 2004. Reserva Natural de Yotoco: su vegetación leñosa. Colombia Forestal No. 17 (1): 75-93.

Cavelier, J., D. Lizcaino \& M. T. Pulido. 2001. Bosques nublados del neotrópico: Colombia. En: M. Kapelle \& A. Brown (eds.). Bosques nublados del neotrópico. Instituto Nacional de Biodiversidad. Santo Domingo de Heredia. Pg. 700.

Cogollo, A. \& J. G. Ramírez. 1997. Estudio sobre la biodiversidad en la Reserva Natural Regional Bajo Cauca-Nechí en el departamento de Antioquia. Fundación Jardín Botánico Joaquín Antonio Uribe de Medellín. Pg. 79.

Cuervo, A. M. 2002. Efecto de la fragmentación de hábitat sobre aves de bosques sub-andinos en los municipios de Amalfí y Anorí. Informe final. Corantioquia. Medellín.

Dueñas A., A. Betancur \& R. Galindo. 2007. Estructura y composición florística de un bosque húmedo tropical del Parque Nacional Natural Catatumbo Barí, Colombia. Colombia Forestal 10 (20): 26-35.

Franco-Rosselli, P., J. Betancur \& J. L. Fernández-Alonso. 1997. Diversidad florística en dos bosques subandinos del sur de Colombia. Caldasia 19 (1-2): 205-234.

Gentry, A.H. 1982. Patterns of neotropical plants species diversity. Evolutionary Biology 15: $1-84$.

Gentry, A. H. 1995. Patterns of diversity and floristic composition in neotropical montane forest. En: S. P. Churchill H. Baslev, E. Forero \& J. L. Lutyn (eds.). Biodiversity and conservartion of 
neotropical montane forests. The New York Botanical Garden. Pp. 103-126.

Gentry, A. H. 2001. Patrones de diversidad y composición florística en los bosques de las montañas neotropicales. En: M. Kapelle \& A. Brown. Bosques nublados del neotropico. Instituto Nacional de Biodiversidad, INBio. Costa Rica.

Gómez, D. 2005. Análisis florístico de los bosques premontanos en el municipio de Anorí (Antioquia). Informe Final. Corantioquia. Medellín. Pg. 150.

Hernández, J. \& H. Sánchez. 1992. Biomas terrestres de Colombia. En: G. Halffter (comp.). La diversidad biológica de Iberoamérica. Acta Zoológica Mexicana. Vol. especial. México.

Instituto Geográfico Agustín Codazzi (IGAC). 1976. Zonas de vida o formaciones vegetales de Colombia. Memoria explicativa sobre Mapa Ecológico. Bogotá.

Isa-Jaum. 2004. Propuesta metodológica de parcelas normalizadas para los inventarios de vegetación. Equipo de investigación Convenio ISAJAUM. Medellín. Pp. 3-10.

Judd, W. S., C. S. Campbell, E. A. Kellog \& S. P. F. Sterens. 1999. Plant Systematics: A phylogenetic approach. Sinauer Assoc. Sunderland. Pg. 464.

Kappelle, M. \& N. Zamora. 1995. Change in woody species richness along an altitudinal gradient in Talamancan montane Quercus forests, Costa Rica. En: Biodiversity of Conservation of Neotropical Montane Forests. The New York Botanical Garden. Bronx, New York. Pp. 53-77.

Lamprecht, H. 1990. Silvicultura en los trópicos. República Federal Alemana. GTZ.

Lema, T. A. 1995. Dasometría. Algunas aproximaciones estadísticas a la medición forestal. Universidad Nacional de Colombia. Medellín. Pg. 401.

López, W., L. Barreto, A. Duque \& F. Moreno. 2006. Composición florística, abundancia y pa- trones de diversidad alfa en dos fragmentos de bosques montanos en la región de los Andes. Informe final. Corantioquia. Medellín. Pg. 87.

Magurran, A. E. 1989. Diversidad ecológica y su medición. Editorial Vedra. Barcelona.

Navarro, J. 2004. Análisis florístico y estructural de los bosques de piedemonte, en el municipio de San Luis de Cubarral (Meta). Tesis de grado. Ingeniería Forestal. Universidad Distrital Francisco José de Caldas.

Rangel-Ch., J. O., A. M. Cleef, S. Salamanca \& C. Ariza. 2004. La vegetación de los bosques y selvas del Tatamá. En: T. van der Hammen, J. O. Rangel-Ch. \& A. M. Cleef (eds.). La cordillera occidental - transecto del Tatamá. Ecoandes 6. J. Crammer. Berlín, Stuttgaart.

Rangel-Ch., J. O. \& A. Velázquez. 1997. Métodos de estudio de la vegetación. En: J. O. RangelCh., P. Lowry \& M. Aguilar. Colombia Diversidad Biótica II. Tipos de vegetación en Colombia. Universidad Nacional de Colombia. Bogotá. Pp. 59-87.

Robert, A. 2003. Simulation of the effect of topography and tree falls on stand dynamics and stand structure of tropical forests. Ecological Modelling 167 (3): 287-303.

Smith, A. R., K. M. Pryer, E. Schuettpelz, P. Korall, H. Schneider \& P. G. Wolf. 2006. A classification for extant ferns. Taxon 55 (3): 705-731.

Tuberquia, D., D. Rodríguez \& C. Gutiérrez. 2000. Informe proyecto educación ambiental basada en inventarios florísticos y prácticas de propagación de especies vegetales del bosque húmedo tropical (Municipios Anorí y Amalfí). Fondo Nacional de Regalías \& Jardín Botánico Joaquín Antonio Uribe. Medellín.

Webster, L. G. 1995. The panorama of neotropical cloud forest. En: S. P. Churchill, H. Baslev, E. Forero \& J. L. Lutyn (eds.). Biodiversity and conservartion of neotropical montane forests. The New York Botanical Garden. Pp. 103-126. 


\section{ANEXOS}

Anexo 1. Listado de especies encontradas en un bosque premontano en el municipio de Amalfi, Antioquia, Colombia, con su familia botánica, la cita de un ejemplar botánico de referencia y el tipo de muestreo empleado para su recolección.

\begin{tabular}{|c|c|c|c|c|c|}
\hline \multirow[b]{2}{*}{ Número } & \multirow[b]{2}{*}{ Nombre científico } & \multirow[b]{2}{*}{ Familia } & \multirow{2}{*}{$\begin{array}{l}\text { *Ejemplar de } \\
\text { referencia }\end{array}$} & \multicolumn{2}{|c|}{ Tipo de recolección } \\
\hline & & & & Transecto & $\begin{array}{l}\text { Muestreo } \\
\text { general }\end{array}$ \\
\hline 1 & Aphelandra boyacensis Leonard & ACANTHACEAE & WAC 1356 & & $x$ \\
\hline 2 & Saurauia brachybotrys Turcz. & ACTINIDIACEAE & WAC 991 & $x$ & $x$ \\
\hline 3 & Viburnum cornifolium Killip \& A.C. Sm. & ADOXACEAE & WAC 999 & $x$ & \\
\hline 4 & Bomarea carderi Mast. & ALSTROEMERIACEAE & WAC 1219 & & $x$ \\
\hline 5 & Alzatea verticillata Ruiz \& Pav. & ALZATEACEAE & WAC 1014 & & $x$ \\
\hline 6 & Tapirira guianensis Aubl. & ANACARDIACEAE & WAC 757 & $x$ & $x$ \\
\hline 7 & Guatteria amplifolia Triana \& Planch. & ANNONACEAE & GT 532 & $x$ & \\
\hline 8 & Guatteria cestrifolia Triana \& Planch. & ANNONACEAE & WAC 663 & $x$ & \\
\hline 9 & Guatteria cf. cargadero Triana \& Planch. & ANNONACEAE & WAC 1355 & $x$ & $x$ \\
\hline 10 & Guatteria lehmannii R.E. Fr. & ANNONACEAE & WAC 1145 & $x$ & \\
\hline 11 & Klarobelia anomala R.E. Fr. Chatrou & ANNONACEAE & WAC 805 & $x$ & \\
\hline 12 & Annona montana Macfad. & ANNONACEAE & GT 521 & & $x$ \\
\hline 13 & Guatteria recurvisepala R. \& Fries & ANNONACEAE & WAC 1042 & & $x$ \\
\hline 14 & Rollinia pittieri Saff. & ANNONACEAE & GT 561 & & $x$ \\
\hline 15 & Xylopia aromatica (Lam.) Mart. & ANNONACEAE & WAC 1211 & & $x$ \\
\hline 16 & Aspidosperma desmanthum Benth. ex Müll. Arg. & APOCYNACEAE & WAC 1444 & $\mathrm{X}$ & $x$ \\
\hline 17 & Fischeria blepharopetala S.F. Blake. & APOCYNACEAE & WAC 1090 & $x$ & \\
\hline 18 & Rauvolfia leptophylla A.S. Rao & APOCYNACEAE & WAC 1208 & $x$ & \\
\hline 19 & Tabernaemontana heterophylla Vahl. & APOCYNACEAE & WAC 1077 & & $x$ \\
\hline 20 & Tassadia obovata Decne. & APOCYNACEAE & WAC 1193 & & $x$ \\
\hline 21 & Ilex cf. danielis Killip \& Cuatrec. & AQUIFOLIACEAE & WAC 1364 & $X$ & \\
\hline 22 & Ilex cf. laurina Kunth & AQUIFOLIACEAE & WAC 1374 & $x$ & \\
\hline 23 & llexsp. & AQUIFOLIACEAE & WAC 917 & $x$ & \\
\hline 24 & Ilex maxima W.J. Hahn & AQUIFOLIACEAE & WAC 1337 & & $x$ \\
\hline 25 & Anthurium cf. caucanum Engl. & ARACEAE & WAC 895 & $x$ & \\
\hline 26 & Anthurium cupreum Engl. & ARACEAE & WAC 1414 & $x$ & $x$ \\
\hline 27 & Anthurium myosuroides (Kunth) Schott & ARACEAE & WAC 846 & $x$ & \\
\hline 28 & Anthurium scandens (Aubl.) Engl. & ARACEAE & GT 527 & $x$ & \\
\hline 29 & Euterpe precatoria Mart. & ARECACEAE & WAC 1445 & $x$ & \\
\hline 30 & Monstera adansonii Schott & ARACEAE & WAC 796 & $X$ & \\
\hline 31 & Monstera dubia (Kunth) Engl. \& K. Krause & ARACEAE & WAC 715 & $x$ & \\
\hline 32 & Monstera obliqua Miq. & ARACEAE & WAC 802 & $x$ & \\
\hline 33 & Philodendron fragrantissimum (Hook.) G. Don & ARACEAE & WAC 727 & $x$ & \\
\hline 34 & Philodendron cf. panamense K. Krause & ARACEAE & WAC 721 & $x$ & \\
\hline 35 & Philodendron sp. & ARACEAE & WAC 728 & $x$ & \\
\hline
\end{tabular}




\begin{tabular}{|c|c|c|c|c|c|}
\hline \multirow[b]{2}{*}{ Número } & \multirow[b]{2}{*}{ Nombre científico } & \multirow[b]{2}{*}{ Familia } & \multirow{2}{*}{$\begin{array}{l}\text { *Ejemplar de } \\
\text { referencia }\end{array}$} & \multicolumn{2}{|c|}{ Tipo de recolección } \\
\hline & & & & Transecto & $\begin{array}{l}\text { Muestreo } \\
\text { general }\end{array}$ \\
\hline 36 & Philodendron strictum G.S. Bunting & ARACEAE & WAC 890 & $x$ & \\
\hline 37 & Stenospermatium aff. spruceanum Schott & ARACEAE & WAC 1458 & $x$ & \\
\hline 38 & Stenospermation andreanum Engl. & ARACEAE & WAC 898 & $x$ & \\
\hline 39 & Philodendron tripartitum (Jacq.) Schott & ARACEAE & WAC 1232 & & $x$ \\
\hline 40 & Xanthosoma daguense Engl. & ARACEAE & WAC 803 & & $x$ \\
\hline 41 & Dendropanax cf. querceti Donn. Sm. & ARALIACEAE & WAC 869 & $x$ & \\
\hline 42 & Dendropanax sp. & ARALIACEAE & WAC 1071 & $x$ & \\
\hline 43 & Schefflera fontiana Cuatrec. & ARALIACEAE & WAC 1257 & $x$ & \\
\hline 44 & Schefflera sp. 3 & ARALIACEAE & GT 461 & $x$ & \\
\hline 45 & Schefflera sp. 1 & ARALIACEAE & WAC 1050 & & $x$ \\
\hline 46 & Schefflera sp.2 & ARALIACEAE & WAC 673 & & $x$ \\
\hline 47 & Aiphanes hirsuta Burret & ARECACEAE & WAC 961 & $x$ & \\
\hline 48 & Geonoma interrupta (Ruiz \& Pav.) Mart. & ARECACEAE & WAC 1329 & $x$ & \\
\hline 49 & Pholidostachys synanthera (Mart.) H.E. Moore & ARECACEAE & WAC 970 & $x$ & \\
\hline 50 & Prestoea acuminata (Willd.) H.E. Moore & ARECACEAE & WAC 972 & $x$ & \\
\hline 51 & Socratea exorrhiza (Mart.) H. Wendl. & ARECACEAE & WAC 808 & $x$ & \\
\hline 52 & $\begin{array}{l}\text { Wettinia fascicularis (Burret) H.E. Moore \& J. } \\
\text { Dransf. }\end{array}$ & ARECACEAE & WAC 1163 & $x$ & \\
\hline 53 & Wettinia kalbreyeri (Burret) R. Bernal & ARECACEAE & WAC 932 & $x$ & \\
\hline 54 & Geonoma undata Klotzsch & ARECACEAE & WAC 1455 & & $x$ \\
\hline 55 & Mikania sp. & ASTERACEAE & WAC 1143 & $x$ & \\
\hline 56 & Paragynoxys corei (Cuatrec.) Cuatrec. & ASTERACEAE & WAC 1045 & $x$ & $x$ \\
\hline 57 & Baccharis nitida (Ruiz \& Pav.) Pers. & ASTERACEAE & WAC 1137a & & $x$ \\
\hline 58 & $\begin{array}{l}\text { Condylidium iresinoides (Kunth) R.M. King \& H. } \\
\text { Rob. }\end{array}$ & ASTERACEAE & WAC 870 & & $x$ \\
\hline 59 & Ichthyothere garcia-barrigae H. Rob. & ASTERACEAE & WAC 1119 & & $x$ \\
\hline 60 & Mikania hookeriana DC. & ASTERACEAE & WAC 1138 & & $x$ \\
\hline 61 & Munnozia senecionidis Benth. & ASTERACEAE & WAC 1139 & & $x$ \\
\hline 62 & Pentacalia trianae (S. Díaz \& S. Obando) Cuatrec. & ASTERACEAE & GT 511 & & $x$ \\
\hline 63 & Piptocoma discolor (Kunth) Pruski & ASTERACEAE & WAC 892 & & $x$ \\
\hline 64 & Vernonanthura patens (Kunth) H. Rob. & ASTERACEAE & GT 556 & & $x$ \\
\hline 65 & Begonia fischeri Schrank & BEGONIACEAE & WAC 1205 & $x$ & \\
\hline 66 & Tabebuia guayacan (Seem.) Hemsl. & BIGNONIACEAE & WAC 1238 & & $x$ \\
\hline 67 & Cordia barbata J. Estrada & BORAGINACEAE & WAC 1141 & & $x$ \\
\hline 68 & Cordia dwyeri Nowicke & BORAGINACEAE & WAC 1412 & & $x$ \\
\hline 69 & Guzmania angustifolia (Baker) Wittm. & BROMELIACEAE & WAC 893 & $x$ & \\
\hline 70 & Guzmania pearcei (Baker) L.B. Sm. & BROMELIACEAE & WAC 871 & $x$ & \\
\hline 71 & Guzmania pungens L.B. Sm. & BROMELIACEAE & WAC 1450 & $x$ & \\
\hline 72 & Pitcairnia bicolor L.B. Sm. \& Read & BROMELIACEAE & WAC 1173 & $x$ & \\
\hline 73 & Guzmania laeta H. Luther & BROMELIACEAE & WAC 877 & & $x$ \\
\hline 74 & Pitcairnia kalbreyeri Baker & BROMELIACEAE & WAC 865 & & $x$ \\
\hline
\end{tabular}




\begin{tabular}{|c|c|c|c|c|c|}
\hline \multirow[b]{2}{*}{ Número } & \multirow[b]{2}{*}{ Nombre científico } & \multirow[b]{2}{*}{ Familia } & \multirow{2}{*}{$\begin{array}{l}\text { *Ejemplar de } \\
\text { referencia }\end{array}$} & \multicolumn{2}{|c|}{ Tipo de recolección } \\
\hline & & & & Transecto & $\begin{array}{l}\text { Muestreo } \\
\text { general }\end{array}$ \\
\hline 75 & $\begin{array}{l}\text { Racinaea steyermarkii (L.B. Sm.) M.A. Spencer \& } \\
\text { L.B. Sm. }\end{array}$ & BROMELIACEAE & WAC 898 & & $x$ \\
\hline 76 & Tillandsia orbicularis L.B. Sm. & BROMELIACEAE & WAC 690 & & $x$ \\
\hline 77 & Vriesea elata (Baker) L.B. Sm. & BROMELIACEAE & WAC 732 & & $x$ \\
\hline 78 & Dacryodes sp. & BURSERACEAE & WAC 1300 & $x$ & \\
\hline 79 & Protium aff. tovarense Pittier & BURSERACEAE & WAC 1004 & & $x$ \\
\hline 80 & Burmeistera cf. microphylla Donn. Sm. & CAMPANULACEAE & WAC 1058 & & $x$ \\
\hline 81 & Centropogon granulosus C. Presl & CAMPANULACEAE & WAC 1335 & & $x$ \\
\hline 82 & Siphocampylus cf. longibracteolatus & CAMPANULACEAE & WAC 1059 & & $x$ \\
\hline 83 & Tontelea attenuata Miers & CELASTRACEAE & WAC 1467 & $x$ & \\
\hline 84 & Maytenus sp. & CELASTRACEAE & WAC 841 & & $x$ \\
\hline 85 & Zinowiewia australis Lundell & CELASTRACEAE & WAC 1171 & & $x$ \\
\hline 86 & Hedyosmum gentryi D’Arcy \& Liesner & CHLORANTHACEAE & WAC 1003 & & $x$ \\
\hline 87 & Hedyosmum racemosum (Ruiz \& Pav.) G. Don & CHLORANTHACEAE & WAC 1366 & & $x$ \\
\hline 88 & Couepia platycalyx Cuatrec. & CHRYSOBALANACEAE & WAC 919 & $x$ & \\
\hline 89 & $\begin{array}{l}\text { Licania octandra (Hoffmanns. ex Roem. \& Schult.) } \\
\text { Kuntze }\end{array}$ & CHRYSOBALANACEAE & WAC 683 & $x$ & \\
\hline 90 & Licania sp. & CHRYSOBALANACEAE & WAC 1362 & $x$ & \\
\hline 91 & Clethra fagifolia Kunth & CLETHRACEAE & WAC 990 & $x$ & \\
\hline 92 & Clethra cf. Ianata M. Martens \& Galeotti & CLETHRACEAE & WAC 1183 & & $x$ \\
\hline 93 & Calophyllum brasiliense Cambess. & CLUSIACEAE & WAC 976 & $x$ & $x$ \\
\hline 94 & Chrysochlamys sp. & CLUSIACEAE & GT 492 & $x$ & \\
\hline 95 & Clusia aff. magnifolia Cuatrec. & CLUSIACEAE & WAC 1008 & $x$ & $x$ \\
\hline 96 & Clusia caicedoi Cuatrec. & CLUSIACEAE & WAC 1006 & $x$ & \\
\hline 97 & Clusia cuneifolia Cuatrec. & CLUSIACEAE & WAC 685 & $x$ & \\
\hline 98 & Clusia cylindrica Hammel & CLUSIACEAE & WAC 1190 & $x$ & $x$ \\
\hline 99 & Clusia lineata (Benth.) Planch. \& Triana & CLUSIACEAE & WAC 1060 & $x$ & \\
\hline 100 & Dystovomita sp. & CLUSIACEAE & WAC 773 & $x$ & $x$ \\
\hline 101 & Marila geminata Cuatrec. & CLUSIACEAE & WAC 1031 & $x$ & $X$ \\
\hline 102 & Tovomita parviflora Cuatrec. & CLUSIACEAE & GT 520 & $x$ & \\
\hline 103 & Tovomita weddelliana Planch. \& Triana & CLUSIACEAE & WAC 1000 & $x$ & \\
\hline 104 & Clusia latipes Planch. \& Triana & CLUSIACEAE & WAC 1186 & & $x$ \\
\hline 105 & Clusia microstemon Planch. \& Triana & CLUSIACEAE & GT 502 & & $x$ \\
\hline 106 & $\begin{array}{l}\text { Clusia schomburgkiana (Planch. \& Triana) Benth. } \\
\text { ex Engl. }\end{array}$ & CLUSIACEAE & WAC 1040 & & $x$ \\
\hline 107 & Commelina sp. & COMMELINACEAE & WAC 1309 & & $x$ \\
\hline 108 & Alsophila cuspidata (Kunze) D.S. Conant & CYATHEACEAE & WAC 867 & $x$ & \\
\hline 109 & Cyathea multiflora Sm. & CYATHEACEAE & WAC 1055 & $x$ & \\
\hline 110 & Cyathea parvula (Jenman) Domin & CYATHEACEAE & WAC 842 & $x$ & \\
\hline 111 & Dicksonia sellowiana Hook. & CYATHEACEAE & WAC 1068 & $x$ & \\
\hline 112 & Asplundia ahlneri Harling & CYCLANTHACEAE & WAC 692 & $x$ & \\
\hline 113 & Cyclanthus bipartitus Poit. ex A. Rich. & CYCLANTHACEAE & WAC 780 & $x$ & \\
\hline
\end{tabular}




\begin{tabular}{|c|c|c|c|c|c|}
\hline \multirow[b]{2}{*}{ Número } & \multirow[b]{2}{*}{ Nombre científico } & \multirow[b]{2}{*}{ Familia } & \multirow{2}{*}{$\begin{array}{c}\text { *Ejemplar de } \\
\text { referencia }\end{array}$} & \multicolumn{2}{|c|}{ Tipo de recolección } \\
\hline & & & & Transecto & $\begin{array}{l}\text { Muestreo } \\
\text { general }\end{array}$ \\
\hline 114 & Dicranopygium sp. & CYCLANTHACEAE & WAC 1308 & $x$ & \\
\hline 115 & Sphaeradenia garciae Harling & CYCLANTHACEAE & WAC 733 & $x$ & \\
\hline 116 & Pteridium arachnoideum (Kaulf.) Maxon & DENNSTAEDTIACEAE & WAC 872 & & $x$ \\
\hline 117 & Tapura colombiana Cuatrec. & DICHAPETALACEAE & WAC 843 & $x$ & \\
\hline 118 & Sloanea brevispina Earle Sm. & ELAEOCARPACEAE & WAC 741 & $x$ & \\
\hline 119 & Cavendishia axillaris A.C. Sm. & ERICACEAE & WAC 1025 & $x$ & \\
\hline 120 & Cavendishia isernii Sleumer & ERICACEAE & WAC 1041 & $x$ & \\
\hline 121 & Orthaea minor (A.C. Sm.) Luteyn & ERICACEAE & WAC 1180 & $x$ & \\
\hline 122 & Psammisia cf. citrina Luteyn \& Sylva & ERICACEAE & WAC 1030 & $x$ & \\
\hline 123 & Satyria cf. breviflora Hoerold & ERICACEAE & WAC 1178 & $x$ & \\
\hline 124 & Bejaria aestuans Mutis ex L. & ERICACEAE & WAC 1005 & & $x$ \\
\hline 125 & Disterigma cryptocalyx A.C. Sm. & ERICACEAE & WAC 1048 & & $x$ \\
\hline 126 & Psammisia ferruginea A.C. Sm. & ERICACEAE & WAC 1215 & & $x$ \\
\hline 127 & Erythroxylum citrifolium A. St.-Hil. & ERYTHROXYLACEAE & WAC 997 & $x$ & $x$ \\
\hline 128 & Alchornea sp. & EUPHORBIACEAE & WAC 734 & $x$ & \\
\hline 129 & Hieronyma oblonga (Tul.) Müll. Arg. & EUPHORBIACEAE & WAC 1078 & $x$ & $x$ \\
\hline 130 & Hyeronima sp. & EUPHORBIACEAE & WAC 845 & $x$ & \\
\hline 131 & Alchornea costaricensis Pax \& K. Hoffm. & EUPHORBIACEAE & WAC 1443 & & $x$ \\
\hline 132 & Croton billbergianus Müll. Arg. & EUPHORBIACEAE & WAC 1410 & & $x$ \\
\hline 133 & Hyeronima macrocarpa Müll. Arg. & EUPHORBIACEAE & WAC 549 & & $x$ \\
\hline 134 & Tetrorchidium robledoanum Cuatrec. & EUPHORBIACEAE & WAC 1399 & & $x$ \\
\hline 135 & Quercus humboldtii Bonpl. & FAGACEAE & WAC 781 & $x$ & $x$ \\
\hline 136 & $\begin{array}{l}\text { Colombobalanus excelsa (Lozano et al.) Nixon \& } \\
\text { Crepet }\end{array}$ & FAGACEAE & WAC 1064 & & $x$ \\
\hline 137 & Tachia parviflora Maguire \& Weaver & GENTIANACEAE & WAC 1172 & $x$ & \\
\hline 138 & Symbolanthus pterocalyx Struwe & GENTIANACEAE & WAC 694 & & $x$ \\
\hline 139 & Besleria fallax C.E. Gonzalez, L.E. Skog \& Amaya & GESNERIACEAE & WAC 1100 & $x$ & $x$ \\
\hline 140 & Besleria fecunda C.V. Morton & GESNERIACEAE & WAC 1130 & $x$ & $x$ \\
\hline 141 & Besleria formosa C.V. Morton & GESNERIACEAE & WAC 788 & $x$ & $x$ \\
\hline 142 & Columnea cf. florida C.V. Morton & GESNERIACEAE & WAC 1209 & $x$ & $x$ \\
\hline 143 & Columnea cf. sanguinea (Pers.) Hanst. & GESNERIACEAE & WAC 1019 & $x$ & $x$ \\
\hline 144 & Alloplectus ichthyoderma Hanst & GESNERIACEAE & WAC 736 & & $x$ \\
\hline 145 & Alloplectus panamensis C.V. Morton. & GESNERIACEAE & WAC 782 & & $x$ \\
\hline 146 & Heliconia burleana Abalo \& G. Morales L. & HELICONIACEAE & WAC 1387 & & $x$ \\
\hline 147 & Billia rosea (Planch. \& Linden) C. Ulloa \& P. Jørg. & HIPPOCASTANACEAE & WAC 1072 & $x$ & $x$ \\
\hline 148 & Hymenophyllum sp. & HYMENOPHYLLACEAE & WAC 1451 & $x$ & \\
\hline 149 & Trichomanes diversifrons (Bory) Mett. ex Sadeb. & HYMENOPHYLLACEAE & WAC 695 & $x$ & \\
\hline 150 & Vismia laevis Triana \& Planch. & HYPERICACEAE & WAC 1146 & & $x$ \\
\hline 151 & Dendrobangia boliviana Rusby & ICACINACEAE & WAC 848 & & $x$ \\
\hline 152 & Discophora guianensis Miers & ICACINACEAE & WAC 1407 & & $x$ \\
\hline
\end{tabular}




\begin{tabular}{|c|c|c|c|c|c|}
\hline \multirow[b]{2}{*}{ Número } & \multirow[b]{2}{*}{ Nombre científico } & \multirow[b]{2}{*}{ Familia } & \multirow{2}{*}{$\begin{array}{l}\text { *Ejemplar de } \\
\text { referencia }\end{array}$} & \multicolumn{2}{|c|}{ Tipo de recolección } \\
\hline & & & & Transecto & $\begin{array}{l}\text { Muestreo } \\
\text { general }\end{array}$ \\
\hline 153 & Indeterminada & INDETERMINADA & WAC 1162 & & $x$ \\
\hline 154 & Alfaroa colombiana Lozano, Hern. Cam. \& Espinal & JUGLANDACEAE & WAC 888 & $x$ & \\
\hline 155 & Lozania mutisiana Schult. & LACISTEMATACEAE & WAC 1018 & & $x$ \\
\hline 156 & Hyptidendron arboreum (Benth.) Harley & LAMIACEAE & WAC 1347 & & $x$ \\
\hline 157 & Aniba coto (Rusby) Kosterm. & LAURACEAE & WAC 1175 & $x$ & $x$ \\
\hline 158 & Aniba puchury-minor (Mart.) Mez & LAURACEAE & WAC 1029 & $x$ & \\
\hline 159 & Aniba taubertiana Mez & LAURACEAE & WAC 1331 & $x$ & \\
\hline 160 & $\begin{array}{l}\text { Beilschmiedia costaricensis (Mez \& Pittier) C.K. } \\
\text { Allen }\end{array}$ & LAURACEAE & WAC 696 & $x$ & \\
\hline 161 & Endlicheria pyriformis (Nees) Mez & LAURACEAE & WAC 783 & $x$ & \\
\hline 162 & Endlicheria sericea Nees & LAURACEAE & GT 481 & $x$ & \\
\hline 163 & Endlicheria rubriflora Mez & LAURACEAE & WAC 738 & $x$ & \\
\hline 164 & Lauraceae sp. 1 & LAURACEAE & WAC 785 & $x$ & \\
\hline 165 & Lauraceae sp. 2 & LAURACEAE & WAC 777 & $x$ & \\
\hline 166 & Lauraceae sp. 3 & LAURACEAE & WAC 875 & $x$ & \\
\hline 167 & Nectandra cufodontisii (0.C. Schmidt) C.K. Allen & LAURACEAE & WAC 1221 & $x$ & \\
\hline 168 & Nectandra membranacea (Ruiz \& Pav.) Nees & LAURACEAE & WAC 873 & $x$ & \\
\hline 169 & Ocotea aciphylla (Nees) Mez & LAURACEAE & WAC 778 & $x$ & \\
\hline 170 & Ocotea aurantiodora (Ruiz \& Pav.) Mez & LAURACEAE & WAC 869 & $x$ & \\
\hline 171 & Ocotea costulata (Nees) Mez & LAURACEAE & WAC 697 & $x$ & \\
\hline 172 & Ocotea leucoxylon (Sw.) Laness. & LAURACEAE & WAC 849 & $x$ & \\
\hline 173 & Ocotea oblonga (Meisn.) Mez & LAURACEAE & WAC 737 & $x$ & \\
\hline 174 & Ocotea puberula (Rich.) Nees & LAURACEAE & WAC 874 & $x$ & \\
\hline 175 & Ocotea smithiana 0 . Schmidt & LAURACEAE & WAC 766 & $x$ & \\
\hline 176 & Persea areolatocostae (C.K. Allen) van der Werff & LAURACEAE & WAC 642 & $x$ & \\
\hline 177 & Persea hexanthera L.E. Kopp & LAURACEAE & WAC 779 & $x$ & \\
\hline 178 & Pleurothyrium glabritepalum van der Werff & LAURACEAE & WAC 981 & $x$ & \\
\hline 179 & Pleurothyrium trianae (Mez) Rohwer & LAURACEAE & WAC 698 & $x$ & \\
\hline 180 & Endlicheria tschudyana (Lasser) Kosterm. & LAURACEAE & WAC 1168 & & $x$ \\
\hline 181 & Nectandra acutifolia (Ruiz \& Pav.) Mez & LAURACEAE & WAC 1259 & & $x$ \\
\hline 182 & Nectandra cuspidata Nees & LAURACEAE & WAC 1207 & & $x$ \\
\hline 183 & Nectandra laurel Klotzsch ex Nees & LAURACEAE & WAC 1147 & & $x$ \\
\hline 184 & Ocotea guianensis Aubl. & LAURACEAE & WAC 714 & & $x$ \\
\hline 185 & Ocotea macropoda (Kunth) Mez & LAURACEAE & WAC 784 & & $x$ \\
\hline 186 & Mutisiopersea chrysophylla (L.E. Kopp) Kosterm. & LAURACEAE & WAC 1016 & & $x$ \\
\hline 187 & Persea cuneata Meisn. & LAURACEAE & WAC 660 & & $x$ \\
\hline 188 & Eschweilera coriacea (DC.) S.A. Mori & LECYTHIDACEAE & WAC 1450 & $x$ & \\
\hline 189 & Eschweilera sessilis A.C. Sm. & LECYTHIDACEAE & WAC 1049 & & $x$ \\
\hline 190 & Andira chigorodensis R.T. Penn. & LEGUMINOSAE & WAC 693 & $x$ & \\
\hline 191 & Dussia lehmannii Harms & LEGUMINOSAE & WAC 1459 & $x$ & $x$ \\
\hline
\end{tabular}




\begin{tabular}{|c|c|c|c|c|c|}
\hline \multirow[b]{2}{*}{ Número } & \multirow[b]{2}{*}{ Nombre científico } & \multirow[b]{2}{*}{ Familia } & \multirow{2}{*}{$\begin{array}{l}\text { *Ejemplar de } \\
\text { referencia }\end{array}$} & \multicolumn{2}{|c|}{ Tipo de recolección } \\
\hline & & & & Transecto & $\begin{array}{l}\text { Muestreo } \\
\text { general }\end{array}$ \\
\hline 192 & Inga cf. auristellae Harms & LEGUMINOSAE & WAC 1357 & $x$ & \\
\hline 193 & Inga cf. leptocarpa T.D. Penn. & LEGUMINOSAE & GT 540 & $x$ & \\
\hline 194 & Inga cinnamomea Spruce ex Benth. Kuntze & LEGUMINOSAE & WAC 1250 & $X$ & $x$ \\
\hline 195 & Inga cocleensis Pittier & LEGUMINOSAE & WAC 1249 & $x$ & $x$ \\
\hline 196 & Inga sp. & LEGUMINOSAE & GT 562 & $x$ & \\
\hline 197 & Macrolobium sp. & LEGUMINOSAE & WAC 1378 & $x$ & \\
\hline 198 & Macrolobium sp. 2 & LEGUMINOSAE & WAC 795 & $x$ & \\
\hline 199 & Parkia nitida Miq. & LEGUMINOSAE & WAC 1039 & $x$ & $x$ \\
\hline 200 & Abarema callejasii Barneby \& Grimes & LEGUMINOSAE & WAC 1017 & & $x$ \\
\hline 201 & Albizia carbonaria Britton & LEGUMINOSAE & WAC 1446 & & $x$ \\
\hline 202 & Inga heterophylla Willd. & LEGUMINOSAE & WAC 1328 & & \\
\hline 203 & Inga marginata Willd. & LEGUMINOSAE & WAC 717 & & $x$ \\
\hline 204 & Ormosia revoluta Rudd & LEGUMINOSAE & WAC 831 & & $x$ \\
\hline 205 & Roucheria columbiana Hallier f. & LINACEAE & WAC 1015 & $x$ & $x$ \\
\hline 206 & Strychnos erichsonii R.H. Schomb. ex Progel & LOGANIACEAE & GT 485 & $x$ & \\
\hline 207 & Lomariopsis vestita E. Fourn. & LOMARIOPSIDACEAE & WAC 1457 & $x$ & \\
\hline 208 & Aetanthus ovalis Rusby & LORANTHACEAE & WAC 699 & & $x$ \\
\hline 209 & Gaiadendron punctatum (Ruiz \& Pav.) G. Don & LORANTHACEAE & WAC 1009 & & $x$ \\
\hline 210 & Oryctanthus occidentalis (L.) Eichler & LORANTHACEAE & WAC 851 & & $x$ \\
\hline 211 & Oryctanthus spicatus (Jacq.) Eichler & LORANTHACEAE & WAC 740 & & $x$ \\
\hline 212 & Phthirusa pyrifolia (Kunth) Eichler & LORANTHACEAE & WAC 1244 & & $x$ \\
\hline 213 & Psittacanthus dilatatus A.C. Sm. & LORANTHACEAE & WAC 1189 & & $x$ \\
\hline 214 & Struthanthus leptostachyus (Kunth) G. Don & LORANTHACEAE & WAC 1032 & & $x$ \\
\hline 215 & Magnolia espinalii (Lozano) Govaerts & MAGNOLIACEAE & WAC 700 & & $x$ \\
\hline 216 & Magnolia yarumalensis (Lozano) Govaerts & MAGNOLIACEAE & WAC 664 & & $x$ \\
\hline 217 & Banisteriopsis elegans (Triana \& Planch.) Sandwith & MALPIGHIACEAE & WAC 1012 & $x$ & $x$ \\
\hline 218 & Hiraea sp. & MALPIGHIACEAE & WAC 852 & $x$ & \\
\hline 219 & Byrsonima garcibarrigae Cuatrec. & MALPIGHIACEAE & WAC 1185 & & $X$ \\
\hline 220 & Byrsonima nemoralis Cuatrec. & MALPIGHIACEAE & WAC 1062 & & $x$ \\
\hline 221 & Bombacoideae sp. 2 & MALVACEAE & WAC 893 & $x$ & \\
\hline 222 & Matisia sp. & MALVACEAE & WAC 652 & $x$ & \\
\hline 223 & $\begin{array}{l}\text { Spirotheca rosea (Seem.) P.E. Gibbs \& W.S. } \\
\text { Alverson }\end{array}$ & MALVACEAE & WAC 1021 & $x$ & \\
\hline 224 & $\begin{array}{l}\text { Matisia intricata (A. Robyns \& S. Nilsson) W.S. } \\
\text { Alverson }\end{array}$ & MALVACEAE & GT 483 & & $x$ \\
\hline 225 & Sida acuta Burm. f. & MALVACEAE & WAC 1070 & & $x$ \\
\hline 226 & Calathea sp. & MARANTACEAE & WAC 1310 & $x$ & \\
\hline 227 & Marcgravia affinis Hemsl. & MARCGRAVIACEAE & GT 471 & $x$ & \\
\hline 228 & $\begin{array}{l}\text { Marcgravia cf. brownei (Triana \& Planch.) } \\
\text { Krug \& Urb. }\end{array}$ & MARCGRAVIACEAE & WAC 1169 & $x$ & \\
\hline 229 & $\begin{array}{l}\text { Marcgraviastrum mixtum (Triana \& Planch.) } \\
\text { Bedell }\end{array}$ & MARCGRAVIACEAE & WAC 1075 & $x$ & \\
\hline 230 & Marcgravia dressleri Giraldo-Cañas & MARCGRAVIACEAE & WAC 1220 & & $X$ \\
\hline
\end{tabular}




\begin{tabular}{|c|c|c|c|c|c|}
\hline \multirow[b]{2}{*}{ Número } & \multirow[b]{2}{*}{ Nombre científico } & \multirow[b]{2}{*}{ Familia } & \multirow{2}{*}{$\begin{array}{l}\text { *Ejemplar de } \\
\text { referencia }\end{array}$} & \multicolumn{2}{|c|}{ Tipo de recolección } \\
\hline & & & & Transecto & $\begin{array}{l}\text { Muestreo } \\
\text { general }\end{array}$ \\
\hline 231 & Allomaieta hirsuta (Gleason) Lozano & MELASTOMATACEAE & WAC 743 & $x$ & \\
\hline 232 & Allomaieta zenufanasana Lozano & MELASTOMATACEAE & WAC 1415 & $x$ & \\
\hline 233 & Blakea quadrangularis Triana & MELASTOMATACEAE & WAC 917 & $x$ & \\
\hline 234 & Conostegia cf. bracteata Triana & MELASTOMATACEAE & WAC 992 & $x$ & \\
\hline 235 & Graffenrieda cf. micrantha (Gleason) L.0. Williams & MELASTOMATACEAE & WAC 1122 & $x$ & \\
\hline 236 & Graffenrieda latifolia subsp. meridensis Wurdack & MELASTOMATACEAE & WAC 1181 & $x$ & \\
\hline 237 & Meriania antioquiensis L. Uribe & MELASTOMATACEAE & WAC 1011 & $x$ & \\
\hline 238 & Meriania Iongifolia (Naudin) Cogn. & MELASTOMATACEAE & WAC 1022 & $x$ & \\
\hline 239 & Miconia costaricensis Cogn. & MELASTOMATACEAE & WAC 1150 & $x$ & $x$ \\
\hline 240 & Miconia dolichorrhyncha Naudin. & MELASTOMATACEAE & WAC 854 & $x$ & \\
\hline 241 & Miconia floribunda (Bonpl.) DC. & MELASTOMATACEAE & WAC 1182 & $x$ & $x$ \\
\hline 242 & Miconia lamprophylla Triana & MELASTOMATACEAE & WAC 996 & $x$ & $x$ \\
\hline 243 & Miconia prasina (Sw.) DC. & MELASTOMATACEAE & WAC 1247 & $x$ & \\
\hline 244 & Miconia punctata (Desr.) D. Don ex DC. & MELASTOMATACEAE & WAC 1180 & $x$ & $x$ \\
\hline 245 & Miconia reducens Triana & MELASTOMATACEAE & WAC 1054 & $x$ & $x$ \\
\hline 246 & Miconia resima Naudin & MELASTOMATACEAE & WAC 742 & $x$ & \\
\hline 247 & Miconia theizans (Bonpl.) Cogn. & MELASTOMATACEAE & WAC 1234 & $x$ & \\
\hline 248 & Topobea inflata Triana & MELASTOMATACEAE & WAC 1352 & $x$ & \\
\hline 249 & Adelobotrys adscendens (Sw.) Triana & MELASTOMATACEAE & WAC 1157 & & $x$ \\
\hline 250 & Adelobotrys sp. & MELASTOMATACEAE & WAC 1069 & & $x$ \\
\hline 251 & Blakea calyptrata Gleason & MELASTOMATACEAE & WAC 1245 & & $x$ \\
\hline 252 & Conostegia montana (Sw.) D. Don ex DC. & MELASTOMATACEAE & WAC 876 & & $x$ \\
\hline 253 & Graffenrieda galeottii (Naudin) L.0. Williams & MELASTOMATACEAE & WAC 787 & & $x$ \\
\hline 254 & Graffenrieda gracilis (Triana) L.0. Williams & MELASTOMATACEAE & WAC 701 & & $x$ \\
\hline 255 & Henriettella aff. trachyphylla Triana & MELASTOMATACEAE & WAC 786 & & $x$ \\
\hline 256 & Miconia dodecandra Cogn. & MELASTOMATACEAE & WAC 1140 & & $x$ \\
\hline 257 & Miconia gracilis Triana & MELASTOMATACEAE & WAC 937 & & $x$ \\
\hline 258 & Miconia mirabilis (Aubl.) L.O.Williams & MELASTOMATACEAE & WAC 681 & & \\
\hline 259 & Miconia serrulata (DC.) Naudin & MELASTOMATACEAE & GT 584 & & $x$ \\
\hline 260 & Miconia stenostachya DC. & MELASTOMATACEAE & WAC 1180 & & $x$ \\
\hline 261 & Salpinga dimorpha (Gleason) Wurdack & MELASTOMATACEAE & WAC 1035 & & $x$ \\
\hline 262 & Tibouchina lepidota (Bonpl.) Baill. & MELASTOMATACEAE & WAC 1038 & & $x$ \\
\hline 263 & Guarea glabra Vahl & MELIACEAE & WAC 1061 & $x$ & \\
\hline 264 & Ruagea glabra Triana \& Planch. & MELIACEAE & WAC 1149 & & $x$ \\
\hline 265 & Trichilia martiana C. DC. & MELIACEAE & GT 470 & & $x$ \\
\hline 266 & Trichilia pallida Sw. & MELIACEAE & WAC 744 & & $x$ \\
\hline 267 & Mendoncia glabrescens Leonard & MENDONCIACEAE & WAC 1010 & $x$ & \\
\hline 268 & Anomospermum reticulatum (Mart.) Eichler & MENISPERMACEAE & WAC 1159 & & $x$ \\
\hline 269 & Mollinedia cf. killipii J.F. Macbr. & MONIMIACEAE & WAC 745 & $x$ & \\
\hline
\end{tabular}




\begin{tabular}{|c|c|c|c|c|c|}
\hline \multirow[b]{2}{*}{ Número } & \multirow[b]{2}{*}{ Nombre científico } & \multirow[b]{2}{*}{ Familia } & \multirow{2}{*}{$\begin{array}{l}\text { *Ejemplar de } \\
\text { referencia }\end{array}$} & \multicolumn{2}{|c|}{ Tipo de recolección } \\
\hline & & & & Transecto & $\begin{array}{l}\text { Muestreo } \\
\text { general }\end{array}$ \\
\hline 270 & Mollinedia tomentosa (Benth.) Tul. & MONIMIACEAE & WAC 703 & & $x$ \\
\hline 271 & Clarisia biflora Ruiz \& Pav. & MORACEAE & WAC 789 & $x$ & $x$ \\
\hline 272 & Ficus americana Aubl. & MORACEAE & WAC 1013 & $x$ & \\
\hline 273 & $\begin{array}{l}\text { Helicostylis tovarensis (Klotzsch \& Karsten ) } \\
\text { C.C.Berg }\end{array}$ & MORACEAE & WAC 1002 & $x$ & \\
\hline 274 & Naucleopsis capirensis C.C. Berg & MORACEAE & WAC 746 & $x$ & \\
\hline 275 & $\begin{array}{l}\text { Perebea guianensis subsp. castilloides (Pittier) } \\
\text { C.C. Berg }\end{array}$ & MORACEAE & WAC 1051 & $x$ & $x$ \\
\hline 276 & Ficus gomelleira Kunth \& C.D. Bouché & MORACEAE & GT 546 & & $x$ \\
\hline 277 & Ficus mutisii Dugand & MORACEAE & WAC 1388 & & $x$ \\
\hline 278 & Ficus trigona L. f. & MORACEAE & WAC 704 & & $x$ \\
\hline 279 & Sorocea sp. & MORACEAE & WAC 856 & & $x$ \\
\hline 280 & Compsoneura aff. capitellata (A. DC.) Warb. & MYRISTICACEAE & WAC 1216 & $x$ & \\
\hline 281 & Virola macrocarpa A.C. Sm. & MYRISTICACEAE & WAC 1046 & $x$ & \\
\hline 282 & Otoba novogranatensis Moldenke & MYRISTICACEAE & WAC 1319 & & $x$ \\
\hline 283 & Cybianthus occigranatensis (Cuatre.) Angostini & MYRSINACEAE & WAC 1043 & $x$ & \\
\hline 284 & Cybianthus schlimii (Hook. f.) G. Agostini & MYRSINACEAE & WAC 732 & $x$ & \\
\hline 285 & Cybianthus venezuelanus Mez & MYRSINACEAE & WAC 1218 & $x$ & \\
\hline 286 & Myrsine coriacea (Sw.) R. Br. ex Roem. \& Schult. & MYRSINACEAE & WAC 1260 & $x$ & \\
\hline 287 & Parathesis sp. & MYRSINACEAE & WAC 1123 & $x$ & \\
\hline 288 & Geissanthus occidentalis Cuatrec. & MYRSINACEAE & WAC 1028 & & $x$ \\
\hline 289 & Myrsine aff. latifolia (Ruiz \& Pav.) Spreng. & MYRSINACEAE & WAC 1260 & & $x$ \\
\hline 290 & Myrsine pellucida (Ruiz \& Pav.) Spreng. & MYRSINACEAE & WAC 1199 & & $x$ \\
\hline 291 & Eugenia biflora DC. & MYRTACEAE & WAC 712 & $x$ & \\
\hline 292 & Myrcia fallax (Rich.) DC. & MYRTACEAE & WAC 1047 & $x$ & \\
\hline 293 & Myrcia paivae 0. Berg & MYRTACEAE & WAC 747 & $x$ & \\
\hline 294 & Myrcia sp. 1 & MYRTACEAE & WAC 1052 & $x$ & \\
\hline 295 & Myrcia sp. 2 & MYRTACEAE & WAC 1222 & $x$ & \\
\hline 296 & Eugenia sp. 1 & MYRTACEAE & & $x$ & \\
\hline 297 & Eugenia sp. 2 & MYRTACEAE & & $x$ & \\
\hline 298 & Neea amplifolia Donn. Sm. & NYCTAGINACEAE & WAC 1435 & $x$ & \\
\hline 299 & Ouratea cf. ferruginea Engl. & OCHNACEAE & WAC 857 & $x$ & \\
\hline 300 & Ludwigia hyssopifolia (G. Don) Exell & ONAGRACEAE & WAC 1204 & & $x$ \\
\hline 301 & Epidendrum paniculatum Ruiz \& Pav. & ORCHIDACEAE & WAC 1179 & $x$ & \\
\hline 302 & Epidendrum sp. 1 & ORCHIDACEAE & WAC 804 & $x$ & \\
\hline 303 & Epidendrum sp. 2 & ORCHIDACEAE & WAC 920 & $x$ & \\
\hline 304 & Epidendrum sp. 3 & ORCHIDACEAE & WAC 931 & $x$ & \\
\hline 305 & Odontoglossum sp. & ORCHIDACEAE & WAC 1212 & $x$ & \\
\hline 306 & Oncidium sp. & ORCHIDACEAE & WAC 705 & $x$ & \\
\hline 307 & Platystele examen-culicum Luer & ORCHIDACEAE & WAC 839 & $x$ & \\
\hline
\end{tabular}




\begin{tabular}{|c|c|c|c|c|c|}
\hline \multirow[b]{2}{*}{ Número } & \multirow[b]{2}{*}{ Nombre científico } & \multirow[b]{2}{*}{ Familia } & \multirow{2}{*}{$\begin{array}{l}\text { *Ejemplar de } \\
\text { referencia }\end{array}$} & \multicolumn{2}{|c|}{ Tipo de recolección } \\
\hline & & & & Transecto & $\begin{array}{l}\text { Muestreo } \\
\text { general }\end{array}$ \\
\hline 308 & Pleurothallis garayana (Ospina) Luer & ORCHIDACEAE & WAC 858 & $x$ & \\
\hline 309 & Stelis sp. & ORCHIDACEAE & WAC 1024 & $x$ & \\
\hline 310 & Oncidium abortivum Rchb. f. & ORCHIDACEAE & WAC 1144 & & $x$ \\
\hline 311 & Pleurothallis sp. & ORCHIDACEAE & WAC 748 & & $x$ \\
\hline 312 & Sobralia sp. & ORCHIDACEAE & GT 445 & & $x$ \\
\hline 313 & Passiflora alnifolia Kunth & PASSIFLORACEAE & WAC 1079 & & $x$ \\
\hline 314 & Phyllanthus attenuatus Miq. & PHYLLANTHACEAE & WAC 993 & & $X$ \\
\hline 315 & Richeria grandis Vahl & PHYLLANTHACEAE & WAC 706 & & $x$ \\
\hline 316 & Phyllonoma ruscifolia Willd. & PHYLLONOMACEAE & WAC 1066 & & $x$ \\
\hline 317 & Picramnia gracilis Tul & PICRAMNIACEAE & WAC 1326 & $x$ & $x$ \\
\hline 318 & Peperomia sp. & PIPERACEAE & WAC 1469 & $x$ & \\
\hline 319 & Piper aequale Vahl & PIPERACEAE & WAC 1151 & $x$ & \\
\hline 320 & Piper archeri Trel. \& Yunck. & PIPERACEAE & WAC 1198 & $x$ & \\
\hline 321 & Piper munchanum C. DC. & PIPERACEAE & WAC 1202 & $x$ & \\
\hline 322 & Peperomia umbellifera Yunck. & PIPERACEAE & WAC 838 & & $x$ \\
\hline 323 & Piper calceolarium C. DC. & PIPERACEAE & WAC 1152 & & $x$ \\
\hline 324 & Piper glanduligerum C. DC. & PIPERACEAE & WAC 1203 & & $x$ \\
\hline 325 & Chusquea aff. scandens Kunth & POACEAE & WAC 1454 & $x$ & \\
\hline 326 & Chasquea aff. purdieana Munro & POACEAE & GT 450 & $x$ & \\
\hline 327 & $\begin{array}{l}\text { Paspalum candidum (Humb. \& Bonpl. ex Flüggé) } \\
\text { Kunth }\end{array}$ & POACEAE & WAC 1261 & $x$ & \\
\hline 328 & Axonopus compressus (Sw.) & POACEAE & WAC 1262 & & $x$ \\
\hline 329 & Paspalum sp. & POACEAE & WAC 1263 & & $x$ \\
\hline 330 & Podocarpus oleifolius (D. Don ex Lamb.) Kuntze & PODOCARPACEAE & WAC 1264 & & $x$ \\
\hline 331 & Securidaca sp. & POLYGALACEAE & WAC 653 & $x$ & \\
\hline 332 & Monnina celastroides (Bonpl.) Chodat & POLYGALACEAE & WAC 1177 & & $x$ \\
\hline 333 & Campyloneurum angustifolium (Sw.) Fée & POLYPODIACEAE & WAC 859 & & $x$ \\
\hline 334 & Panopsis mucronata Cuatrec. & PROTEACEAE & GT 537 & $x$ & \\
\hline 335 & Roupala montana Aubl. & PROTEACEAE & WAC 707 & & $x$ \\
\hline 336 & Quiina sp. & QUIINACEAE & WAC 836 & $x$ & \\
\hline 337 & Sterigmapetalum colombianum Monach. & RHIZOPHORACEAE & WAC 1161 & $x$ & $x$ \\
\hline 338 & Prunus sp. & ROSACEAE & WAC 711 & $x$ & \\
\hline 339 & Coussarea paniculata (Vahl) Standl. & RUBIACEAE & WAC 638 & $x$ & \\
\hline 340 & Elaeagia pastoensis L.E. Mora & RUBIACEAE & WAC 708 & $x$ & $x$ \\
\hline 341 & Faramea glandulosa Poepp. \& Endl. & RUBIACEAE & WAC 1081 & $x$ & \\
\hline 342 & Faramea lehmannii Standl. & RUBIACEAE & WAC 844 & $x$ & \\
\hline 343 & Faramea occidentalis (L.) A. Rich. & RUBIACEAE & WAC 1196 & $x$ & \\
\hline 344 & Faramea oraria Standl. ex Steyerm & RUBIACEAE & GT 525 & $x$ & \\
\hline 345 & Faramea parvula Standl. & RUBIACEAE & DT 946 & $x$ & \\
\hline 346 & Hippotis brevipes Spruce ex K. Schum. & RUBIACEAE & WAC 1313 & $X$ & $x$ \\
\hline
\end{tabular}




\begin{tabular}{|c|c|c|c|c|c|}
\hline \multirow[b]{2}{*}{ Número } & \multirow[b]{2}{*}{ Nombre científico } & \multirow[b]{2}{*}{ Familia } & \multirow{2}{*}{$\begin{array}{l}\text { *Ejemplar de } \\
\text { referencia }\end{array}$} & \multicolumn{2}{|c|}{ Tipo de recolección } \\
\hline & & & & Transecto & $\begin{array}{l}\text { Muestreo } \\
\text { general }\end{array}$ \\
\hline 347 & Hoffmannia cf. pauciflora Standl. & RUBIACEAE & WAC 770 & $x$ & \\
\hline 348 & Ladenbergia macrocarpa (Vahl) Klotzsch & RUBIACEAE & WAC 994 & $x$ & $x$ \\
\hline 349 & Ladenbergia muzonensis (Goudot) Standl. & RUBIACEAE & WAC 882 & $x$ & $x$ \\
\hline 350 & Notopleura longissima Bremek. & RUBIACEAE & GT 513 & $x$ & \\
\hline 351 & Palicourea angustifolia Kunth & RUBIACEAE & WAC 1065 & $x$ & \\
\hline 352 & Palicourea cf. acetosoides Wernham & RUBIACEAE & WAC 960 & $x$ & \\
\hline 353 & Palicourea cf. calophlebia Standl. & RUBIACEAE & WAC 1074 & $x$ & \\
\hline 354 & Palicourea sp. & RUBIACEAE & WAC 776 & $x$ & \\
\hline 355 & Posoqueria latifolia (Rudge) Roem. \& Schult. & RUBIACEAE & GT 536 & $x$ & \\
\hline 356 & Psychotria allenii Standl. & RUBIACEAE & WAC 835 & $x$ & \\
\hline 357 & Psychotria brachiata Sw. & RUBIACEAE & WAC 1073 & $x$ & \\
\hline 358 & Psychotria poeppigiana Müll. Arg. & RUBIACEAE & WAC 998 & $x$ & \\
\hline 359 & Rudgea cf. colombiana Standl. & RUBIACEAE & WAC 1242 & $x$ & \\
\hline 360 & Amaioua guianensis Aubl. & RUBIACEAE & WAC 860 & & $x$ \\
\hline 361 & Coccocypselum 2hirsutum Bartl. ex DC. & RUBIACEAE & WAC 1241 & & $x$ \\
\hline 362 & Guettarda crispiflora Vahl & RUBIACEAE & WAC 1251 & & $x$ \\
\hline 363 & $\begin{array}{l}\text { Palicourea rigidifolia (Dwyer \& M.V. Hayden) } \\
\text { Dwyer }\end{array}$ & RUBIACEAE & WAC 833 & & $x$ \\
\hline 364 & Palicourea thermydri J.H. Kirkbr. & RUBIACEAE & WAC 1158 & & $x$ \\
\hline 365 & $\begin{array}{l}\text { Psychotria galeottiana (M. Martens) C.M. Taylor } \\
\text { \& Lorence }\end{array}$ & RUBIACEAE & WAC 861 & & $x$ \\
\hline 366 & Psychotria reflexiramea Standl. & RUBIACEAE & WAC 750 & & $x$ \\
\hline 367 & Sabicea cana Hook. f. & RUBIACEAE & WAC 1001 & & $x$ \\
\hline 368 & Zanthoxylum sp. & RUTACEAE & WAC 879 & $x$ & \\
\hline 369 & Hortia brasiliana Vand. ex DC. & RUTACEAE & WAC 793 & & $x$ \\
\hline 370 & Zanthoxylum melanostictum Schltdl. \& Cham & RUTACEAE & WAC 1027 & & $x$ \\
\hline 371 & Meliosma glossophylla Cuatrec. & SABIACEAE & WAC 862 & $x$ & \\
\hline 372 & Banara guianensis Aubl. & SALICACEAE & WAC 1023 & & $x$ \\
\hline 373 & Casearia arborea (Rich.) Urb. & SALICACEAE & WAC 1164 & & $x$ \\
\hline 374 & Casearia cajambrensis Cuatrec. & SALICACEAE & WAC 1214 & & $x$ \\
\hline 375 & Casearia grandiflora Cambess. & SALICACEAE & WAC 847 & & $x$ \\
\hline 376 & Casearia javitensis Kunth & SALICACEAE & WAC 735 & & $x$ \\
\hline 377 & Neoptychocarpus chocoensis A.H. Gentry \& Forero & SALICACEAE & WAC 868 & & $x$ \\
\hline 378 & Allophyllus sp. & SAPINDACEAE & WAC 794 & $x$ & \\
\hline 379 & Matayba arborescens (Aubl.) Radlk. & SAPINDACEAE & WAC 880 & $x$ & \\
\hline 380 & Paullinia pachycarpa Benth. & SAPINDACEAE & GT 571 & $x$ & $x$ \\
\hline 381 & Serjania aff. rhombea Radlk. & SAPINDACEAE & WAC 1109 & $x$ & \\
\hline 382 & Talisia sp. & SAPINDACEAE & WAC 863 & $x$ & \\
\hline 383 & Cupania americana $\mathrm{L}$. & SAPINDACEAE & WAC 1258 & & $x$ \\
\hline 384 & Paullinia clathrata Radlk. & SAPINDACEAE & GT 530 & & $x$ \\
\hline 385 & Chrysophyllum argenteum Jacq. & SAPOTACEAE & WAC 724 & $x$ & \\
\hline
\end{tabular}




\begin{tabular}{|c|c|c|c|c|c|}
\hline \multirow[b]{2}{*}{ Número } & \multirow[b]{2}{*}{ Nombre científico } & \multirow[b]{2}{*}{ Familia } & \multirow{2}{*}{$\begin{array}{l}\text { *Ejemplar de } \\
\text { referencia }\end{array}$} & \multicolumn{2}{|c|}{ Tipo de recolección } \\
\hline & & & & Transecto & $\begin{array}{l}\text { Muestreo } \\
\text { general }\end{array}$ \\
\hline 386 & $\begin{array}{l}\text { Chrysophyllum cf. colombianum (Aubrév.) T.D. } \\
\text { Penn }\end{array}$ & SAPOTACEAE & WAC 918 & $x$ & \\
\hline 387 & Chrysophyllum prieurii A. DC. & SAPOTACEAE & WAC 1057 & $x$ & \\
\hline 388 & Micropholis crotonoides (Pierre) Pierre & SAPOTACEAE & WAC 665 & $x$ & $x$ \\
\hline 389 & Pouteria cf. baehniana Monach. & SAPOTACEAE & WAC 831 & $x$ & \\
\hline 390 & Ecclinusa bullata T.D. Penn. & SAPOTACEAE & GT 501 & & $x$ \\
\hline 391 & Schlegelia monachinoi Moldenke & SCHLEGELIACEAE & WAC 840 & $x$ & \\
\hline 392 & Escobedia grandiflora (L. f.) Kuntze & SCROPHULARIACEAE & WAC 1082 & & $x$ \\
\hline 393 & Picrolemma huberi Ducke & SIMAROUBACEAE & WAC 1411 & & $x$ \\
\hline 394 & Siparuna cf. subscandens A.C. Sm. & SIPARUNACEAE & WAC 855 & $x$ & \\
\hline 395 & Siparuna aspera (Ruiz \& Pav.) A. DC. & SIPARUNACEAE & WAC 1246 & & $x$ \\
\hline 396 & Siparuna conica S.S. Renner \& Hausner & SIPARUNACEAE & WAC 1210 & & $x$ \\
\hline 397 & Smilax siphilitica Humb. \& Bonpl. ex Willd. & SMILACACEAE & WAC 864 & $x$ & \\
\hline 398 & Juanulloa ochracea Cuatrec. & SOLANACEAE & WAC 709 & $x$ & \\
\hline 399 & Solanum thelopodium Sendtn. & SOLANACEAE & WAC 1167 & $x$ & \\
\hline 400 & Cestrum sp. & SOLANACEAE & GT 538 & & $x$ \\
\hline 401 & Solanum aturense Dunal & SOLANACEAE & WAC 1166 & & $x$ \\
\hline 402 & Turpinia occidentalis (Sw.) G. Don & STAPHYLLEACEAE & WAC 837 & & $x$ \\
\hline 403 & Styrax sp. & STYRACACEAE & WAC 881 & $x$ & \\
\hline 404 & Styrax macrocalyx Perkins & STYRACACEAE & WAC 1465 & & $x$ \\
\hline 405 & Gordonia fruticosa (Schrad.) H. Keng & THEACEAE & WAC 798 & $x$ & \\
\hline 406 & Ternstroemia macrocarpa Triana \& Planch. & THEACEAE & WAC 980 & $x$ & \\
\hline 407 & Pourouma minor Benoist & URTICACEAE & WAC 1392 & $x$ & \\
\hline 408 & Cecropia angustifolia & URTICACEAE & WAC 1156 & & $x$ \\
\hline 409 & Cecropia sp. & URTICACEAE & WAC 1460 & & $x$ \\
\hline 410 & Cecropia telenitida Cuatrec. & URTICACEAE & WAC 691 & & $x$ \\
\hline 411 & Phenax hirtus (Sw.) Wedd. & URTICACEAE & WAC 1248 & & $x$ \\
\hline 412 & Pourouma bicolor Mart. & URTICACEAE & WAC 731 & & $x$ \\
\hline 413 & Urera baccifera (L.) Gaudich. ex Wedd. & URTICACEAE & WAC 865 & & $x$ \\
\hline 414 & Valeriana sp. & VALERIANACEAE & WAC 961 & $x$ & \\
\hline 415 & Aegiphila falcata Donn. Sm & VERBENACEAE & WAC 1037 & & $x$ \\
\hline 416 & Aegiphila sp. & VERBENACEAE & WAC 883 & & $x$ \\
\hline 417 & Dendrophthora amalfiensis Kuijt & VISCACEAE & WAC 710 & & $x$ \\
\hline 418 & Erisma sp. & VOCHYSIACEAE & WAC 797 & $x$ & \\
\hline 419 & Qualea sp. & VOCHYSIACEAE & WAC 832 & $x$ & \\
\hline 420 & Vochysia aff. aurantiaca Stafleu & VOCHYSIACEAE & GT 490 & $x$ & $x$ \\
\hline 421 & Renealmia foliifera Standl. & ZINGIBERACEAE & WAC 1327 & & $\mathrm{X}$ \\
\hline
\end{tabular}

*WAC.: William Ariza Cortés.

*GT. Germán Téllez.

*DT.: Dino Tuberquia. 
Anexo. 2. Listado de las veinte especies con mayor IVI encontradas en un bosque premontano en el municipio de Amalfi, Antioquia. Incluye abundancia, frecuencia y área basal.

\begin{tabular}{|c|c|c|c|c|c|c|c|}
\hline Especie & $\begin{array}{l}\text { Abundancia } \\
\text { absoluta }\end{array}$ & $\begin{array}{l}\text { Abundancia } \\
\text { relativa }\end{array}$ & $\begin{array}{l}\text { Frecuencia } \\
\text { absoluta }\end{array}$ & $\begin{array}{l}\text { Frecuencia } \\
\text { relativa }\end{array}$ & $\begin{array}{l}\text { Área basal } \\
\text { absoluta }\end{array}$ & $\begin{array}{l}\text { Área basal } \\
\text { relativa }\end{array}$ & IVI \\
\hline Quercus humboldtii Bonpl. & 4 & 0.008 & 0.2 & $0.34 \%$ & 0.720 & $12.59 \%$ & $13.77 \%$ \\
\hline Protium aff. tovarense Pittier & 22 & 0.046 & 0.8 & $1.36 \%$ & 0.223 & $3.90 \%$ & $9.87 \%$ \\
\hline Chrysophyllum prieurii A. DC. & 3 & 0.006 & 0.6 & $1.02 \%$ & 0.461 & $8.06 \%$ & $9.71 \%$ \\
\hline $\begin{array}{l}\text { Wettinia fascicularis (Burret) H.E. Moore \& } \\
\text { J. Dransf. }\end{array}$ & 25 & 0.052 & 1 & $1.70 \%$ & 0.150 & $2.62 \%$ & $9.56 \%$ \\
\hline Roucheria columbiana Hallier f. & 19 & 0.040 & 1 & $1.70 \%$ & 0.196 & $3.42 \%$ & $9.11 \%$ \\
\hline Dacryodes sp. & 3 & 0.006 & 0.2 & $0.34 \%$ & 0.370 & $6.46 \%$ & $7.43 \%$ \\
\hline Terstroemia macrocarpa Triana \& Planch. & 4 & 0.008 & 0.6 & $1.02 \%$ & 0.299 & $5.22 \%$ & $7.08 \%$ \\
\hline Vochysia aff. aurantiaca aurantiaca Stafleu & 6 & 0.013 & 0.6 & $1.02 \%$ & 0.214 & $3.75 \%$ & $6.02 \%$ \\
\hline Compsoneura aff. capitellata (A. DC.) Warb. & 12 & 0.025 & 0.8 & $1.36 \%$ & 0.112 & $1.96 \%$ & $5.84 \%$ \\
\hline Tovomita weddelliana Planch. \& Triana & 10 & 0.021 & 0.8 & $1.36 \%$ & 0.128 & $2.25 \%$ & $5.70 \%$ \\
\hline $\begin{array}{l}\text { Graffenrieda latifolia subsp. meridensis } \\
\text { Wurdack }\end{array}$ & 11 & 0.023 & 0.8 & $1.36 \%$ & 0.106 & $1.85 \%$ & $5.52 \%$ \\
\hline Micropholis crotonoides (Pierre) Pierre & 4 & 0.008 & 0.6 & $1.02 \%$ & 0.194 & $3.39 \%$ & $5.25 \%$ \\
\hline Miconia punctata (Desr.) D. Don ex DC. & 14 & 0.029 & 0.8 & $1.36 \%$ & 0.031 & $0.55 \%$ & $4.84 \%$ \\
\hline Cyathea multiflora Sm. & 12 & 0.025 & 0.8 & $1.36 \%$ & 0.050 & $0.88 \%$ & $4.76 \%$ \\
\hline Wettinia kalbreyeri (Burret) R. Bernal & 8 & 0.017 & 0.8 & $1.36 \%$ & 0.090 & $1.58 \%$ & $4.62 \%$ \\
\hline Quiinasp. & 3 & 0.006 & 0.6 & $1.02 \%$ & 0.154 & $2.70 \%$ & $4.35 \%$ \\
\hline Aniba coto (Rusby) Kosterm. & 6 & 0.013 & 0.6 & $1.02 \%$ & 0.100 & $1.75 \%$ & $4.03 \%$ \\
\hline Clusia cf. magnifolia Cuatrec. & 11 & 0.023 & 0.8 & $1.36 \%$ & 0.017 & $0.30 \%$ & $3.97 \%$ \\
\hline Miconia lamprophylla Triana & 11 & 0.023 & 0.8 & $1.36 \%$ & 0.002 & $0.04 \%$ & $3.71 \%$ \\
\hline Erythroxylum citrifolium A. St.-Hil. & 7 & 0.015 & 0.6 & $1.02 \%$ & 0.068 & $1.20 \%$ & $3.68 \%$ \\
\hline
\end{tabular}

